\title{
Blind Identification of Multipath Channels: A Parametric Subspace Approach
}

\author{
Lisa Perros-Meilhac, Éric Moulines, Member, IEEE, Karim Abed-Meraim, Pascal Chevalier, and \\ Pierre Duhamel, Fellow, IEEE
}

\begin{abstract}
In this paper, blind identification of single-input multiple-output (SIMO) systems using second-order statistics (SOS) only is considered. Using the assumption of a specular multipath channel, we investigate a parametric variant of the so-called subspace method. Nonparametric subspace-based methods require a precise estimation of the model order; overestimation of the model order leads to inconsistent channel estimates. We show that the parametric subspace method gives consistent channel estimates when only an upper bound of the channel order is known. A new algorithm, which exploits parametric information on the channel structure, is presented. A statistical performance analysis of the proposed parametric subspace criterion is presented; limited Monte Carlo experiments show that the proposed algorithm is second-order optimal for a large class of channels.
\end{abstract}

\section{INTRODUCTION}

$\mathbf{I}$ $\mathrm{N}$ MOBILE or high-frequency (HF) radiocommunication contexts, signals currently propagate from an emitter to a receiver through different paths, due to reflection, diffraction, and scattering on physical objects in the environment (mobile communications) or to reflections on several ionospherical layers (HF transmissions).

We focus on "noncooperative blind" techniques, meaning that the channel estimation is achieved based only on the channel outputs without resorting to training data, which is necessary for passive listening applications but also of great interest in many other situations.

For ten years, many methods have been developed to blindly identify single-input multiple-output (SIMO) systems from the second-order statistics (SOS) of the data (see [29] and the reference therein). An important class of blind SOS-SIMO algorithms are based on the so-called subspace technique. A distinctive advantage of the subspace algorithms is to be "deterministic" as the channel coefficients can be estimated without errors at the infinite signal-to-noise ratio (SNR) limit.

\footnotetext{
Manuscript received August 5, 1999; revised November 1, 2000. L. Perros-Meilhac was supported in part by the CNRS under Grant 550027 and Thomson-CSF Communications. The associate editor coordinating the review of this paper and approving it for publication was Prof. Michail K. Tsatsanis.

L. Perros-Meilhac, É. Moulines, and K. Abed-Meraim are with Image and Signal Processing Department, Ecole Nationale Supérieure des Télécommunications, Paris, France (e-mail: meilhac@tsi.enst.fr; moulines@tsi.enst.fr; abed@tsi.enst.fr).

P. Chevalier is with Thomson-CSF Communications, TCC/BSR/TBR/TSI, Gennevilliers, France (e-mail: pascal.chevalier@tcc.thomson-csf.com).

P. Duhamel is with the Laboratoire de Signaux et Systmes, Gif sur Yvette,

France (e-mail: pierre.duhamel@1ss.supelec.fr).

Publisher Item Identifier S 1053-587X(01)01427-1.
}

However, as shown by many authors, subspace methods have some severe drawbacks. In particular, they are highly sensitive to modeling errors: an overdetermination of the channel length leads to inconsistent estimates. In practical situations, the exact channel order should be estimated from the data, which is proved to be a rather involved task (moreover, most of the time, the concept of channel order is ill defined [20]; see also [10] and [34] for some possible adaptations of subspace ideas leading to robust algorithms).

In addition, subspace methods in their original formulation do not take into account the a priori information that is, most of the time, available in digital transmission scenarios, such as the knowledge of the pulse shape or prior knowledge on the structure of the propagation channel. It is a well-known fact in statistical estimation that improved performance can be expected if one can put enough constraint on the model structure, leading to a reduction in the number of "free" parameters. Modifications aiming at incorporating in the blind subspace method the knowledge of the pulse shape generate the reduction of the number of unknown parameters, thus reducing the overall estimation variance [5]-[7], [18], [22], [27].

The introduction of the propagation channel structure in the subspace method is the main purpose of this paper. In many applications, the propagation channel can be modeled as a specular channel with a finite number of rays, each one being characterized by its delay, its complex attenuation, and its direction of arrival. The delay spread may vary from few symbols (GSM link) to more than a decade of symbols (HF link) or several decades of chips (UMTS link). Specular channel is a typical situation for which the "direct" parameterization in terms of the impulse response coefficients is inefficient. With a parametric channel model, the blind identification problem reduces to the blind estimation of the channel parameters (attenuations, relative delays, spatial signatures).

This problem has received considerable attention over the past few years. Most existing methods rely on preliminary estimates of the impulse response (see, e.g., [31]; joint angle and delay estimation is considered, e.g., in [6], [26], and [32]). In the (SOS-SIMO) blind context, however, these two-step methods are "nonrobust" to channel overestimation, being based on inconsistent estimates. Recently, direct parametric SOS-SIMO methods have been proposed in [9], [12], [28], and [33]. On the other hand, blind estimation of time delay has also been considered in [2], [13], and [16]; the methods proposed in these contributions are different from those developed in this contribution, being based on cyclo correlation and cyclic spectrum. 
We propose a parametric version of the subspace method [21], exploiting a specular model of the propagation channel and the prior knowledge of the pulse shape filter. If the pulse shape filter is not precisely known (e.g., in a passive listening context), solutions proposed in [22] can be adapted. The proposed algorithm proceeds in "one-pass," exploiting directly the parameterization (and not in a post-processing stage), leading to the so-called "parametric" subspace approach (in contrast with the "unstructured" plain subspace algorithm). The key result of this contribution is that parametric subspace methods lead to consistent estimates of the channel parameters as soon as an upper bound of the channel order is known (which is, of course, weaker and, in practice, much more relevant than the assumption of a known order). The price to be paid (as in most "parametric subspace methods") is that the minimization is no longer a convex problem. A multidimensional search algorithm is required, the typical dimension of the problem being the number of path delays. Some results developed here have been introduced in [24] and [25].

The paper is organized as follows. The assumptions made on the data model are given in Section II. Section III reviews the so-called "plain" subspace method and presents (Theorem 1) a new characterization of the set of solutions (extension of [1, Theorem 6]) when the channel order is overestimated. In Section IV, a new parametric subspace algorithm is presented, and the consistency of this algorithm is addressed (Theorem 2), with a special emphasis on the practical situation where the channel order is overestimated. In Section V, the statistical performance analysis of the parametric subspace method is assessed. It is shown, under mild conditions on the probability distribution of the input signal, that the parameter estimates are consistent and asymptotically normal. The asymptotic covariance matrix is expressed in closed form. Finally, in Section VI, some numerical comparisons of the asymptotic covariance with the Cramér-Rao lower bound (for Gaussian input) are provided. Possible extensions to improve the performance are suggested.

\section{MODEL AND ASSUMPTIONS}

Under standard assumptions (linear modulation over a linear time-invariant channel), the baseband representation of the continuous signal received on $q$ sensors may be expressed as

$$
\mathbf{x}(t)=\sum_{l=-\infty}^{\infty} \mathbf{h}(t-l T) s(l)+\mathbf{n}(t)
$$

where $\{s(k)\}$ are the transmitted symbols, and $T$ is the symbol period; the observation vector $\mathrm{x}(t)$ and the composite channel impulse response $\mathbf{h}(t)$ are defined as $q \times 1$ column vectors

$$
\begin{aligned}
\mathbf{x}(t) & =\left[x_{(1)}(t), \cdots, x_{(q)}(t)\right]^{T} \\
\mathbf{h}(t) & =\left[h_{(1)}(t), \cdots, h_{(q)}(t)\right]^{T}
\end{aligned}
$$

$\mathbf{n}(t)$ is defined similarly to $\mathbf{x}(t)$. The following is assumed in the sequel.

H1) The source signal $\{s(k)\}$ is a sequence of independent and identically distributed (i.i.d.) random variables with zero-mean, unit variance.
H2) $\mathbf{n}(t)$ is a stationary temporally and spatially white Gaussian noise, with zero-mean and second-order moments $\mathbb{E}\left[\mathbf{n}(t) \mathbf{n}(t)^{H}\right]=\sigma^{2} \mathbf{I}_{q}$ and $\mathbb{E}\left[\mathbf{n}(t) \mathbf{n}(t)^{T}\right]=0$. Moreover, $\{\mathbf{n}(t)\}_{t \in \mathbb{R}}$ is independent from $\{s(k)\}_{k \in \mathbb{Z}}$. The vector $\mathbf{h}(t)$ includes the effects of the pulse-shaping filter denoted $g(t)$ and of the propagation channel associated with each sensor. The channel is assumed to be specular, i.e., the sum of a small number of rays. Each ray is parameterized by a delay $\tau$ and a spatial attenuation factor $\mathbf{a}$. The major assumptions we make on the multipath scenario are listed as follows.

M1) The propagation channel is a finite superposition of rays.

M2) The delay spread is finite, $\tau_{\max }<\infty$, and an upperbound of the delay spread is known.

M3) The signal is narrowband with respect to the array aperture.

M4) The pulse-shaping filter $g(t)$ is known and has finite support $g(t)=0$ for $t \notin\left[0, L_{g} T[\right.$.

M5) The channel is stationary over the length of the observation interval. Doppler shift and residual carriers are neglected.

Let $d$ denote the number of delays. Under the above stated assumptions, the response is expressed as

$$
\mathbf{h}(t)=\sum_{k=1}^{d} \mathbf{a}_{k} g\left(t-\tau_{k}\right), \quad \tau_{1}<\cdots<\tau_{d}
$$

where $\tau_{k}$ and $\mathbf{a}_{k}$ are the unknown delay and spatial signature associated with the $k$ th path. If the multipath is directional, $\mathbf{a}_{k}=$ $\beta_{k} \mathbf{a}\left(\theta_{k}\right)$, where $\mathbf{a}(\theta)$ is the array response to a point source for direction $\theta$, and $\beta$ is the fading factor. In this paper, we process the array response $\mathbf{a}_{k}$ as an arbitrary vector of dimension $q$. The observation vector $\mathrm{x}(t)$ is sampled with period $T / p\left(p \in \mathbb{N}^{*}\right)$. For $1 \leq i \leq p$, we define

$$
\begin{aligned}
& \mathbf{h}^{(i)}(k)=\mathbf{h}((i-1) T / p+k T) \\
& \mathbf{x}^{(i)}(k)=\mathbf{x}((i-1) T / p+k T) .
\end{aligned}
$$

By combining spatial and temporal diversity, we form a SIMO linear system with $r=p q$ outputs

$$
\begin{aligned}
& \underline{\mathbf{h}}(k)=\left[\mathbf{h}^{(1)}(k)^{T}, \cdots, \mathbf{h}^{(p)}(k)^{T}\right]_{r \times 1}^{T} \\
& \underline{\mathbf{x}}(k)=\left[\mathbf{x}^{(1)}(k)^{T}, \cdots, \mathbf{x}^{(p)}(k)\right]_{r \times 1}^{T} .
\end{aligned}
$$

Under assumptions M2 and M4, the channel response is a causal finite impulse response system of duration $L T$ with $L=L_{g}+\left\lceil\left(\tau_{d}-\tau_{1}\right) / T\right\rceil$. Then, we can define the $r$ SIMO response $\underline{\mathbf{h}}(z)=\sum_{k=0}^{L} \underline{\mathbf{h}}(k) z^{-k}$, and we may express $\underline{\mathbf{x}}(k)$ as

$$
\underline{\mathbf{x}}(k)=[\underline{\mathbf{h}}(z)] s(k)+\underline{\mathbf{n}}(k) .
$$

In the sequel, it is also convenient to consider the vector $\overrightarrow{\mathbf{h}}=\left[\underline{\mathbf{h}}(0)^{T}, \cdots, \underline{\mathbf{h}}(L)^{T}\right]^{T}$ of dimension $r(L+1)$ obtained by stacking the coefficients of the polynomial vector $\underline{\mathbf{h}}(z)$. Similarly, stacking $(K+1)$ successive samples of the array response, we may rewrite (3) as

$$
\overrightarrow{\mathbf{x}}(k)=\mathcal{T}_{K}(\mathbf{h}) \mathbf{s}(k)+\overrightarrow{\mathbf{n}}(k)
$$


where $\overrightarrow{\mathbf{x}}(k)=\left[\underline{\mathbf{x}}(k)^{T}, \cdots, \underline{\mathbf{x}}(k-K)^{T}\right]_{r(K+1)}^{T}$, $\mathbf{s}(k)=[s(k), \cdots, s(k-K-L)]_{K+L+1}^{T}$, and

$$
\mathcal{T}_{K}(\mathbf{h}) \triangleq \overbrace{\left[\begin{array}{cccc}
\underline{\mathbf{h}}(0) & \cdots & \underline{\mathbf{h}}(L) & 0 \\
0 & \underline{\mathbf{h}}(0) & \cdots & \underline{\mathbf{h}}(L)
\end{array}\right]}^{K+L+1}\} r(K+1) .
$$

For the developments that follow, it is convenient to define the $z$-transform of the oversampled channel $\mathbf{h}_{T / p}(z)=\sum_{k=-\infty}^{\infty} \mathbf{h}(k T / p) z^{-k}$. It is easily seen that

$$
\mathbf{h}_{T / p}(z)=\sum_{i=1}^{p} \mathbf{h}^{(i)}\left(z^{p}\right) z^{-i}
$$

where $\mathbf{h}^{(i)}(z)=\sum_{k=-\infty}^{\infty} \mathbf{h}^{(i)}(k) z^{-k}$. From (2), $\mathbf{h}_{T / p}(z)$ may be written as

$$
\mathbf{h}_{T / p}(z)=\sum_{k=1}^{d} \mathbf{a}_{k} g_{T / p}\left(\tau_{k} ; z\right)
$$

where $g_{T / p}(\tau ; z) \triangleq \sum_{n} g(n(T / p)-\tau) z^{-n}$ is the $z$-transform of the pulse shape filter sampled with rate $T / p$ and phase $\tau$.

\section{SUBSPACE METHOD}

In this section, we first give a description of the subspace algorithm, assuming that the channel order is known. Since this condition is rarely met, we then consider the case where the channel order is overestimated, and we propose an extended version of the subspace identifiability conditions presented in $[1$, th. 6]. This extended version forms the basis of the structured subspace algorithm presented in the following section.

For now

$v_{0} \quad$ true value of the parameter of interest;

$\hat{v} \quad$ estimated value;

$v$ current value.

For any matrix $\mathbf{A}$, we denote by $\operatorname{Span}(\mathbf{A})$ the linear space spanned by the columns of $\mathbf{A}$ and by $\operatorname{Null}(\mathbf{A})$ the kernel of $\mathbf{A}$. Under assumptions $\mathrm{H} 1$ and $\mathrm{H} 2$, the covariance matrix of the received signal $\overrightarrow{\mathrm{x}}(k)$ may be written as

$$
\mathbf{R}_{0}=\mathbb{E}\left[\overrightarrow{\mathbf{x}}(k) \overrightarrow{\mathbf{x}}(k)^{H}\right]=\mathcal{T}_{K}\left(\mathbf{h}_{0}\right) \mathcal{T}_{K}\left(\mathbf{h}_{0}\right)^{H}+\sigma^{2} \mathbf{I} .
$$

It is well known (see, e.g., [19]) that when $\underline{\mathbf{h}}_{0}(z)$ is irreducible, i.e., $\underline{\mathbf{h}}_{0}(z) \neq 0$ for all $z$, then for all $K \geq L_{0}, \operatorname{rank}\left(\mathcal{T}_{K}\left(\mathbf{h}_{0}\right)\right)=$ $K+L_{0}+1$. Hence, the eigendecomposition of the covariance matrix $\mathbf{R}_{0}$ reads

$$
\mathbf{R}_{0}=\mathbf{F} \operatorname{diag}\left(\lambda_{1}+\sigma^{2}, \cdots, \lambda_{K+L_{0}+1}+\sigma^{2}\right) \mathbf{F}^{H}+\sigma^{2} \mathbf{G G}^{H}
$$

where $\left(\sqrt{\lambda}_{1}, \cdots, \sqrt{\lambda}_{K+L_{0}+1}\right)$ are the singular values of $\mathcal{T}_{K}\left(\mathbf{h}_{0}\right)$, and $\mathbf{F}$ contains the associated left singular vectors. The columns of $\mathbf{G}$ form an (arbitrary) orthogonal basis spanning the orthogonal complement of $\operatorname{Span}(\mathbf{F})$, which is referred to as the noise subspace. Denote $\boldsymbol{\Pi}_{0}=\mathbf{G G}^{H}$ as the orthogonal projector onto the noise subspace of $\mathbf{R}_{0}$. By (7), the noise subspace corresponds to the left null space of $\mathcal{T}_{K}\left(\mathbf{h}_{0}\right)$; thus

$$
\operatorname{Span}\left(\boldsymbol{\Pi}_{0}\right)=\operatorname{Null}\left(\mathcal{T}_{K}\left(\mathbf{h}_{0}\right)^{H}\right) .
$$

In addition, it has been shown in [1] and [21] that $\overrightarrow{\mathbf{h}}_{0}$ is up to an irrelevant scalar factor the unique solution of the linear equation $\Pi_{0} \mathcal{T}_{K}(\mathbf{h})=0$ under the "order constraint" $\operatorname{deg} \underline{\mathbf{h}}(z)=L_{0}$. Moreover, since the matrix $\mathcal{T}_{K}(\mathbf{h})$ depends linearly on $\overrightarrow{\mathbf{h}}$, we may write

$$
\operatorname{Vec}\left(\boldsymbol{\Pi} \mathcal{T}_{K}(\mathbf{h})\right)=\mathcal{D}_{L}(\boldsymbol{\Pi})^{H} \overrightarrow{\mathbf{h}}
$$

where $\mathcal{D}_{L}(\boldsymbol{\Pi})=\left[\mathcal{T}_{L}\left(\boldsymbol{\pi}_{1}\right), \cdots, \mathcal{T}_{L}\left(\boldsymbol{\pi}_{r(K+1)}\right)\right]$ is a $r(L+1) \times r(K+1)(K+L+1)$ block-Toeplitz matrix with $\boldsymbol{\Pi}=\left[\overrightarrow{\boldsymbol{\pi}}_{1}, \cdots, \overrightarrow{\boldsymbol{\pi}}_{r(K+1)}\right]$, i.e., $\overrightarrow{\boldsymbol{\pi}}_{i}$ is the $i$ th column of II. Equation (8) implies that $\operatorname{Span}\left(\overrightarrow{\mathbf{h}}_{0}\right)=\operatorname{Null}\left(\mathcal{D}_{L_{0}}\left(\boldsymbol{\Pi}_{0}\right)^{H}\right)$.

Thus, if $\hat{L}=L_{0}$, the response is identified up to a multiplicative constant. The case where the channel order is underestimated $\hat{L}<L_{0}$ has been studied in [20]. This point is particularly relevant when applying the unstructured subspace method to channels with small leading and trailing terms. As we will see below, the situation is rather different in the parametric case because the channel structure restores identifiability when the channel order is overestimated. In this case, it seems (as shown in the simulations) to be a safe practice to overestimate the channel length. We will now study the impact of such overestimation on the plain subspace identifiability.

Assume that $\hat{L}$, which is the estimated channel order, is larger than $L_{0}$, i.e., $\hat{L} \geq L_{0}$. It has been shown in [1] that the solutions of the linear equations

$$
\mathcal{D}_{\hat{L}}\left(\boldsymbol{\Pi}_{0}\right)^{H} \overrightarrow{\mathbf{h}}=0
$$

are given by $\underline{\hat{\mathbf{h}}}(z)=c(z) \underline{\mathbf{h}}_{0}(z)$, where $c(z)$ is an arbitrary scalar polynomial of degree less than $\hat{L}-L_{0}$. This result, however, gives only a partial answer to the overestimation problem because (10) implicitly assumes that $\boldsymbol{\Pi}_{0}$ is known. In practice, for an estimated value $\hat{L}$, we form $\mathbf{G}_{\hat{L}}$ by taking an (arbitrary) orthogonal subset of $r(K+1)-(K+\hat{L}+1)$ vectors in the null subspace. If $\hat{L}>L_{0}$, the subspace spanned by $\mathbf{G}_{\hat{L}}$ is "strictly" included in the noise subspace, i.e., $\Pi_{\hat{L}} \Pi_{0}=\Pi_{0} \Pi_{\hat{L}}=\Pi_{\hat{L}} \neq$ $\boldsymbol{\Pi}_{0}$, where $\boldsymbol{\Pi}_{\hat{L}}=\mathbf{G}_{\hat{L}} \mathbf{G}_{\hat{L}}^{H}$. Theorem 1 below shows that the solutions of the equations $\mathcal{D}_{\hat{L}}\left(\boldsymbol{\Pi}_{\hat{L}}\right)^{H} \overrightarrow{\mathbf{h}}=0$ are also given by $\underline{\hat{\mathbf{h}}}(z)=c(z) \underline{\mathbf{h}}_{0}(z)$. This new result is better suited to the practical situation of interest.

Theorem 1: Let $\underline{\mathbf{h}}_{0}(z)$ be a $r \times 1$ irreducible polynomial vector $\left(\underline{\mathbf{h}}_{0}(z) \neq 0 \forall z\right)$ of degree $L_{0}$, and let $\underline{\hat{\mathbf{h}}}(z) \neq 0$ be a $r \times 1$ polynomial vector of degree $\hat{L} \geq L_{0}$. Let $K \geq \hat{L}$ and let $\Pi_{L_{0}}$ and $\Pi_{\hat{L}}$ be two matrices $(r(K+1) \times r(K+1))$ verifying

$$
\begin{aligned}
\operatorname{Span}\left(\boldsymbol{\Pi}_{L_{0}}\right) & =\operatorname{Null}\left(\mathcal{T}_{K}\left(\mathbf{h}_{0}\right)^{H}\right) \\
\operatorname{Span}\left(\boldsymbol{\Pi}_{\hat{L}}\right) & \subset \operatorname{Span}\left(\boldsymbol{\Pi}_{L_{0}}\right) \\
\operatorname{Rank}\left(\boldsymbol{\Pi}_{\hat{L}}\right) & =r(K+1)-(K+\hat{L}+1) .
\end{aligned}
$$

Then

$$
\Pi_{\hat{L}} \mathcal{T}_{K}(\hat{\mathbf{h}})=0 \Leftrightarrow \underline{\hat{\mathbf{h}}}(z)=c(z) \underline{\mathbf{h}}_{0}(z)
$$

where $c(z)$ is a scalar polynomial of degree $\hat{L}-L_{0}$.

Proof is given in Appendix A. Thus, if the system is overmodeled, then the subspace method leads to an inconsistent estimate as $c(z)$ is arbitrary. 
TABLE I

OutLine OF THE PARAMETRIC SUbSPACE Algorithm

1. Choose $\hat{L} \geq L_{g}+\Delta \tau_{\max }$ where $\Delta \tau_{\max }$ is an upper bound of the relative delay between the paths.

2. Estimate the covariance matrix $\hat{\mathbf{R}}$ (eq. (15)) and calculate $\hat{\Pi}_{\hat{L}}$, the $r(K+1)-(K+\hat{L}+1)$ smallest eigenvectors of $\hat{\mathbf{R}}$. Form the matrix $\mathbf{Q}_{\hat{L}}(\hat{\mathbf{R}})$ from $\hat{\boldsymbol{\Pi}}_{\hat{L}}$.

3. Obtain an estimate $\dot{d}$ of the number of path delays (see e.g. [35][11]).

4. Estimate the set of delays: $\hat{\tau}=\arg \min _{\tau} \breve{J}_{\hat{L}, \hat{d}}(\boldsymbol{\tau})$. The number of delays being generally small, this minimization problem may be achieved a global multidimensional search. We propose the following iterative process.

- Assume $d=1$. Obtain $\hat{\tau}_{1}$ minimizing $\breve{J}_{\hat{L}, d}\left(\tau_{1}\right)$

- Assume $d=2$. Obtain an estimate $\hat{\tau}_{2}$ of $\tau_{2}$, by minimizing $\breve{J}_{\hat{L} d}\left(\left[\tau_{1}, \tau_{2}\right]\right)$ in function of $\tau_{2}$ keeping $\tau_{1}$ fixed equal to $\hat{\tau}_{1}$ obtained in first step. Next, obtain a new estimate of $\left\{\tau_{1}, \tau_{2}\right\}$ by doing a local multidimensional search using $\left\{\hat{\tau}_{1}, \hat{\tau}_{2}\right\}$ previously obtained as initial value.

- This process is iterated until $d$ is equal to $\hat{d}$.

5. Obtain directly an estimate of $\mathbf{a}_{0}$ as $\hat{\mathbf{a}}=\operatorname{vp}_{\min }\left(\mathbf{G}_{\hat{L}, \hat{d}}(\hat{\tau})^{H} \mathbf{Q}_{\hat{L}}(\hat{\mathbf{R}}) \mathbf{G}_{\hat{L}, \hat{d}}(\hat{\boldsymbol{\tau}})\right)$ where $\operatorname{vp}_{\min }(A)$ denotes the eigenvector corresponding to the minimal eigenvalue of matrix $A$.

In practice, $N(N-K \geq r(K+1))$ samples $\mathrm{x}(k)$ are observed, and an estimate $\hat{\Pi}$ of $\boldsymbol{\Pi}_{\hat{L}}$ may be constructed from the following estimate of the covariance matrix:

$$
\hat{\mathbf{R}}=\frac{1}{N-K} \sum_{k=K+1}^{N} \overrightarrow{\mathbf{x}}(k) \overrightarrow{\mathbf{x}}(k)^{H}
$$

by taking the eigenvectors associated wiht the $r(K+1)-(K+$ $\hat{L}+1$ ) smallest eigenvalues of $\hat{\mathbf{R}}$. We solve (9) in a least-square sense, minimizing

$$
J_{\hat{L}, \hat{\mathbf{\Pi}}}(\overrightarrow{\mathbf{h}})=\overrightarrow{\mathbf{h}}^{H} \mathbf{Q}_{\hat{L}}(\hat{\mathbf{R}}) \overrightarrow{\mathbf{h}}
$$

where $\mathbf{Q}_{\hat{L}}(\hat{\mathbf{R}})=\mathcal{D}_{\hat{L}}(\hat{\mathbf{\Pi}}) \mathcal{D}_{\hat{L}}(\hat{\mathbf{\Pi}})^{H}$, under the nontriviality constraint $\|\overrightarrow{\mathbf{h}}\|=1$.

\section{PARAMETRIC SUbSPace Method}

\section{A. A Parametric Subspace Algorithm}

Under assumptions M1 to M5, a d-ray specular channel of order $L, \underline{\mathbf{h}}(z)$ may be expressed as [see (6)], $\mathbf{h}_{T / p}(z)=\sum_{k=1}^{d} \mathbf{a}_{k} g_{T / p}\left(\tau_{k} ; z\right)$, and the associated vector $\overrightarrow{\mathbf{h}}$ reads $\overrightarrow{\mathbf{h}}=\mathbf{G}_{L, d}(\boldsymbol{\tau}) \mathbf{a}$, where $\mathbf{a}=\left[\mathbf{a}_{1}^{T} \cdots \mathbf{a}_{d}^{T}\right]_{q . d \times 1}^{T}$ and $\mathbf{G}_{L, d}(\boldsymbol{\tau})=\left(\mathcal{G}(\tau) \otimes \mathbf{I}_{q}\right) . \otimes$ is the Kronecker product, and $\mathcal{G}(\boldsymbol{\tau})$ is a $p(L+1) \times d$ matrix defined as

$$
\begin{aligned}
& \mathcal{G}(\tau)= \\
& \left(\begin{array}{ccc}
g\left(0-\tau_{1}\right) & \cdots & g\left(0-\tau_{d}\right) \\
g\left(\frac{T}{p}-\tau_{1}\right) & \cdots & g\left(\frac{T}{p}-\tau_{d}\right) \\
\vdots & & \vdots \\
g\left((L+1) T-\frac{T}{p}-\tau_{1}\right) & \cdots & g\left((L+1) T-\frac{T}{p}-\tau_{d}\right)
\end{array}\right) .
\end{aligned}
$$

Let $\hat{d} \geq d_{0}$ be the estimated number of delays and $\hat{L} \geq L_{0}$ be the estimated channel order. The parametric subspace estimation consists of minimizing in $(\tau, \mathbf{a})$ the following criterion:

$$
J_{\hat{L}, \hat{d}}(\boldsymbol{\tau}, \mathbf{a})=\mathbf{a}^{H} \mathbf{G}_{\hat{L}, \hat{d}}(\tau)^{H} \mathbf{Q}_{\hat{L}}(\hat{\mathbf{R}}) \mathbf{G}_{\hat{L}, \hat{d}}(\boldsymbol{\tau}) \mathbf{a}
$$

under the constraints $\|\mathbf{a}\| \neq 0$ and $\tau_{i} \neq \tau_{j}$ for $i \neq j$. Several constraints are possible; for example, $\mathbf{e}_{1}^{T} \mathbf{a}=1\left(\mathbf{e}_{1}=\right.$ $\left[\begin{array}{lll}10 & \cdots & 0\end{array}\right]^{T}$ ) is imposed in [25].

Under the constraint $\|\mathbf{a}\|=1$, the minimum in $\mathbf{a}$ of (17) for fixed $\tau$ is the normalized eigenvector corresponding to the minimum eigenvalue of matrix $\mathbf{G}_{\hat{L}, \hat{d}}(\boldsymbol{\tau})^{H} \mathbf{Q}_{\hat{L}}(\hat{\mathbf{R}}) \mathbf{G}_{\hat{L}, \hat{d}}(\tau)$. Thus, the criterion (17) reduces in $\mathbf{a}$ to

$$
\tilde{J}_{\hat{L}, \hat{d}}(\boldsymbol{\tau})=\lambda_{\min }\left(\mathbf{G}_{\hat{L}, \hat{d}}(\boldsymbol{\tau})^{H} \mathbf{Q}_{\hat{L}}(\hat{\mathbf{R}}) \mathbf{G}_{\hat{L}, \hat{d}}(\boldsymbol{\tau})\right)
$$

where $\lambda_{\min }(\mathbf{A})$ denotes the minimum eigenvalue of matrix A. We obtain an estimate $\hat{\boldsymbol{\tau}}$ of $\boldsymbol{\tau}_{0}$ minimizing the reduced criterion (18) of $\hat{d}$ parameters under the constraint $\tau_{i} \neq \tau_{j}$. Then, an estimate $\hat{\mathbf{a}}$ of $\mathbf{a}_{0}$ can be obtained directly as the eigenvector corresponding to the minimum eigenvalue of $\mathbf{G}_{\hat{L}, \hat{d}}(\hat{\tau})^{H} \mathbf{Q}_{\hat{L}}(\hat{\mathbf{R}}) \mathbf{G}_{\hat{L}, \hat{d}}(\hat{\tau})$.

Note that when two or more components of the vector $\tau$ are identical, then the rank of the matrix $\mathbf{G}_{\hat{L}, \hat{d}}(\tau)$ degenerates, and the function $\tilde{J}_{\hat{L}, \hat{d}}(\tau)$ is zero. To avoid these ill-formed solutions, we force the constraint $\tau_{i} \neq \tau_{j}$ in the criterion by using, instead of (18), the following normalized criterion:

$$
\breve{J}_{\hat{L}, \hat{d}}(\boldsymbol{\tau})=\frac{\lambda_{\min }\left(\mathbf{G}_{\hat{L}, \hat{d}}(\boldsymbol{\tau})^{H} \mathbf{Q}_{\hat{L}}(\hat{\mathbf{R}}) \mathbf{G}_{\hat{L}, \hat{d}}(\boldsymbol{\tau})\right)}{\lambda_{\min }^{\alpha}\left(\mathbf{G}_{\hat{L}, \hat{d}}(\boldsymbol{\tau})^{H} \mathbf{G}_{\hat{L}, \hat{d}}(\boldsymbol{\tau})\right)}
$$

which is nonzero when matrix $\mathbf{G}_{\hat{L}, \hat{d}}(\boldsymbol{\tau})$ is singular. Taking $\alpha=$ 1 guarantees that the criterion remains bounded in the neighborhood of the spurious singular solutions. Taking $\alpha>1$ forces the criterion to $+\infty$ in that neighborhood. In practice, it appears that $\alpha=1$ is a good choice. Perhaps surprisingly, it is shown in Section V that this normalization does not influence the limiting variance of the estimator, i.e., the normalization does not "asymptotically" affect the position of the minima of the function nor the "shape" (the curvature) of the criterion in the neighborhood of these minima.

The steps of the proposed algorithm are summarized in Table I. Theorem 2 shows that under stated assumptions, the estimators $(\hat{\boldsymbol{\tau}}, \hat{\mathbf{a}})$ are consistent, even when the channel order $\hat{L}$ and the number of delays $\hat{d}$ are overestimated. 


\section{B. Identifiability of the Parametric Model with Channel Order Overestimation}

A multipath propagation channel is characterized by a number of rays $d$, a set $\mathcal{D}$ of $d$ distinct delays $\mathcal{D}=\left\{\tau_{1}, \cdots, \tau_{d}\right\}$ $\left(\tau_{i} \neq \tau_{j}\right.$ for $\left.i \neq j\right)$, and a set of $d$ spatial attenuation factors $\left\{\mathbf{a}_{1}, \cdots, \mathbf{a}_{d}\right\}\left(\mathbf{a}_{i} \neq 0,1 \leq i \leq d\right)$. We use the following notation:

$$
\mathcal{M}=\left\{d,\left\{\tau_{1}, \cdots, \tau_{d}\right\},\left\{\mathbf{a}_{1}, \cdots, \mathbf{a}_{d}\right\}\right\}
$$

Moreover, we define for every $k \in\{1, \cdots, d\}$ the set

$$
\mathcal{C}_{k}(\mathcal{M})=\left\{\tau_{i} \in \mathcal{M} ; \tau_{i}=\tau_{k}+n_{i, k} T, n_{i, k} \in \mathbb{Z}, \tau_{k} \in \mathcal{M}\right\}
$$

i.e., the set of delays $\tau_{i} \in \mathcal{M}$ that differ from $\tau_{k} \in \mathcal{M}$ by an integer multiple of the symbol period. Note that by construction, $\tau_{k} \in \mathcal{C}_{k}(\mathcal{M})$. Finally, we assume that the following property (commented further) is verified. [For a set $\mathbf{A}, \operatorname{card}(\mathbf{A})$ denotes the cardinal of $\mathbf{A}$.]

Proposition 1 (P1): For any $d$-uplets $\left\{\tau_{1}, \tau_{2}, \cdots, \tau_{d}\right\}$ such that $\tau_{i} \neq \tau_{j}$ for $i \neq j$ and such that for all $(n, l) \in \mathbb{N}^{2}$

$$
\operatorname{card}\left(\{ \tau _ { 1 } , \cdots , \tau _ { d } \} \cap \left[n T / p,(n+l+1) T / p[) \leq p L_{g}+l\right.\right.
$$

the matrix $\mathbf{G}_{L, d}\left(\tau_{1}, \cdots, \tau_{d}\right)$ is full rank.

Theorem 2: Let $\mathcal{M}_{0}$ and $\hat{\mathcal{M}}$ be two multipath channels, and let $\mathbf{h}_{0(T / p)}(z)=\sum_{k=1}^{d_{0}} \mathbf{a}_{0 k} g_{T / p}\left(\tau_{0 k} ; z\right)$ and $\hat{\mathbf{h}}_{T / p}(z)=\sum_{k=1}^{\hat{d}} \hat{\mathbf{a}}_{k} g_{T / p}\left(\hat{\tau}_{k} ; z\right)$. Assume that

$$
\hat{\mathbf{h}}_{T / p}(z)=c\left(z^{p}\right) \mathbf{h}_{\mathrm{O}(T / p)}(z)
$$

where $c(z)=\sum_{n=0}^{M} c_{n} z^{-n}$ is a scalar polynomial of degree $M \geq 0$. Assume, in addition, that

I1) $q \geq \max _{k} \operatorname{card}\left(\mathcal{C}_{k}\left(\mathcal{M}_{0}\right)\right)$;

I2) $\operatorname{Rank}\left(\left[\mathbf{a}_{01} \cdots \mathbf{a}_{0 d_{0}}\right]_{q \times d}\right)=\min (q, d)$;

I3) $p L_{g} \geq \max \left(0,\left(d_{0}-\min \left(q, d_{0}\right)-p+1\right)\right) M+d_{0}-$ $\min \left(q, d_{0}\right)+1+\hat{d}$

I4) $d_{0} \leq \hat{d}<2 d_{0}-\delta \leq 2 d_{0}$ where $\delta=$ $\max _{n \in \mathbb{N} *} \operatorname{card}\left\{\left(\tau_{0 i}, \tau_{0 j}\right) \in \mathcal{M}_{0} ; \tau_{0 i}=\tau_{0 j}+n T\right\} ;$

then $c(z)=c_{m} z^{-m} m \in\{0, \cdots, M\}$, and

for $1 \leq k \leq d_{0}$

for $d_{0}<k \leq \hat{d}$

$$
\begin{aligned}
\hat{\tau}_{k} & =\tau_{0 k}+m T \\
\hat{\mathbf{a}}_{k} & =r_{m} \mathbf{a}_{0 k}
\end{aligned}
$$

$$
\hat{\mathbf{a}}_{k}=0 .
$$

The proof is presented in Appendix B. We have seen in Section III that an overestimation of the channel order in the subspace method introduces a polynomial indetermination, which is denoted $c(z)$, i.e., $\underline{\hat{\mathbf{h}}}(z)=c(z) \underline{\mathbf{h}}_{0}(z)$. From (5), this is equivalent to $\hat{\mathbf{h}}_{T / p}(z)=c\left(z^{p}\right) \mathbf{h}_{0(T / p)}(z)$. This theorem means that by imposing the specular structure, the intrinsic subspace indetermination is raised to an irrelevant (and unavoidable in the blind context) translation factor $c(z)=c_{m} z^{-m}$. Thus, $\underline{\hat{\mathbf{h}}}(z)=$ $c_{m} z^{-m} \underline{\mathbf{h}}_{0}(z)$, meaning that up to a multiplicative constant and an unidentifiable offset (equal to an integer number periods), the parametric subspace estimate is consistent. We also have a consistent estimation of the channel parameters.

Comments: In most scenarios, $\max _{k} \operatorname{card}\left(\mathcal{C}_{k}\left(\mathcal{M}_{0}\right)\right)=1$, i.e., the differential delays are not integer multiple of the symbol period. In such case, assumption I1 always holds. Assumption I1 indicates that when the differential delay between two rays of the true response is a multiple of the period symbol, we need spatial diversity to identify these delays. Indeed, note that $g_{T / p}(\tau ; z) z^{-s p}=g_{T / p}(\tau+s T ; z)$ and $c\left(z^{p}\right) g_{T / p}(\tau ; z)=$ $\sum_{s=0}^{M} c_{s} g_{T / p}(\tau+s T ; z)$, where $c\left(z^{p}\right)=\sum_{s=0}^{M} c_{s} z^{-s p}$. Thus, by multiplying the true response by a polynomial scalar $c(z)$, we create a new parametric response having $M d_{0}$ "spurious" rays. The differential delay between spurious rays and the associated "true" ray is an integer multiple of the symbol period. Hence, in this case, a true and a "spurious" ray may correspond to the same time and then cannot be separated using only temporal diversity. Spatial diversity allows us to separate them.

Assumption I4 shows that we can obtain a consistent estimate of the channel parameters, even when the number of rays is overestimated. In most scenarios, $\delta=0$ (see the first remark), and the number of delays can be overestimated by a factor less than two.

Assumption I2 is classical and means that we have distinct angles and unambiguous space manifold. (See [32] on the effective rank of $\left[\begin{array}{lll}\mathbf{a}_{01} & \cdots & \mathbf{a}_{0 d_{0}}\end{array}\right]$ ).

As assumption I3 is very technical, we propose to derive it for some particular cases. First, if $q \geq d_{0}$ (which is the most often considered situation in the literature), then I3 may be replaced by $p L_{g} \geq \hat{d}+1$. Note that in this case, the system remains identifiable, regardless of the channel order overestimation factor $M$. Now, let us take $d_{0} \geq q$ and, more precisely, $d_{0} \geq q+p-1$; then, assumption I3 may be replaced by $p L_{g} \geq\left(d_{0}-q+1\right)(M+1)-M p+\hat{d}$. Thus, perhaps surprisingly, if the channel order is overestimated by a factor compatible with this condition, then it is also possible to identify more rays than sensors. In particular, if $M=0$ (i.e., the channel order is known), then $\mathrm{I} 3$ becomes $p L_{g} \geq d_{0}-q+1+\hat{d}$.

Assumption $\mathrm{I} 3$ assumes that the property $\mathrm{P} 1$ is verified, i.e., that when the number of delays $\left\{\tau_{1}, \tau_{2}, \cdots, \tau_{d}\right\}$ falling in a time interval $[n T / p,(n+l) T / p$ [ is "compatible" with the degree of $g_{T / p}(z)$, then $\mathbf{G}_{L, d}\left(\tau_{1}, \cdots, \tau_{d}\right)$ is full rank. $\mathbf{P 1}$ is generally theoricaly verified, but in practice, performance depends on the condition number of the matrix $\mathbf{G}_{L, d}(\tau)$. We propose to study this point by simulation, as in [31]. We consider $d$ rays equispaced in time between 0 and $10 T$ such that $d$ is the maximum number of delays verifying (20). The modulation waveform is a raised-cosine pulse with excess bandwidth $\alpha$, truncated to zero outside the interval $\left[-B, B\left[\left(L_{g}=2 B-1\right)\right.\right.$. We observe the singular value of the matrix $\mathbf{G}_{L, d}(\boldsymbol{\tau})$ as a function of $p$ (see Fig. 1), $B$ (see Fig. 2) and $\alpha$ (see Fig. 3).

In Figs. 1 and 2, one can see that for $p \geq 2$ and $B>1$, the large singular values are all the same. Thus, in a "bandlimited" context, we cannot tolerate more rays by increasing the oversampling factor $p$ or the order of the pulse shape filter $L_{g}$ (assumption I 3 should thus be interpreted carefully). Fig. 3 shows that the resolution power depends on the excess bandwidth. For a given $\alpha$, one can defined the effective value of $p L_{g}$ (in function of the noise power). 


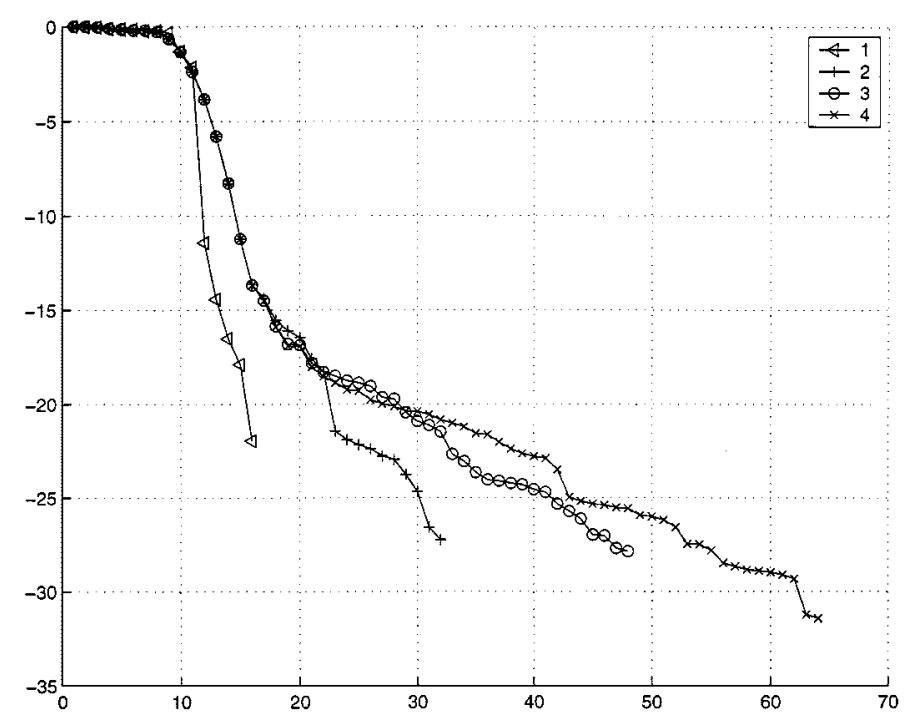

Fig. 1. Singular values of $\mathbf{G}_{L, d}(\boldsymbol{\tau})$ for various oversampling factor $p, B=3$, $\alpha=0.25$.

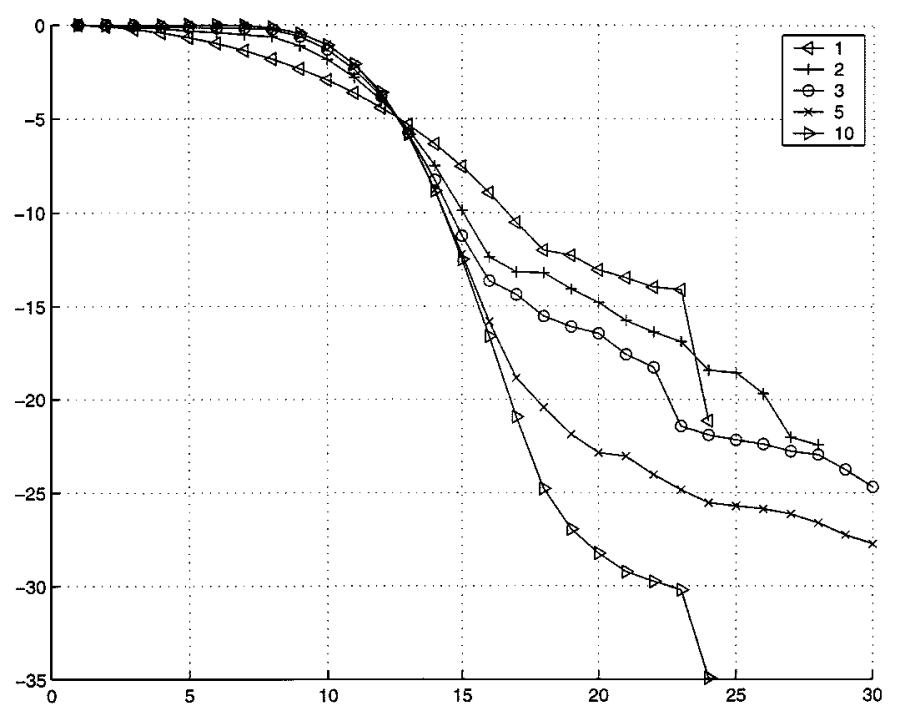

Fig. 2. Singular values of $\mathbf{G}_{L, d}(\boldsymbol{\tau})$ for various half-pulse shape length factor $B, p=2, \alpha=0.25$.

\section{Asymptotic PERformanCE ANALYsis}

In this section, we establish the asymptotic distribution of the parametric subspace estimator and derive an explicit expression for the covariance matrix of its estimation errors.

Theorem 3: The sequence of estimates $\hat{\boldsymbol{\tau}}$ is asymptotically normal with mean $\tau_{0}$ and covariance matrix $\mathcal{C}\left(\tau_{0}, \sigma^{2}\right)$

$$
\sqrt{N}\left(\hat{\tau}-\tau_{0}\right) \rightarrow \mathcal{L} \mathcal{N}\left(0, \mathcal{C}\left(\tau_{0}, \sigma^{2}\right)\right)
$$

$$
\begin{aligned}
& {\left[\mathcal{C}\left(\boldsymbol{\tau}_{0}, \sigma^{2}\right)\right]_{i, j}} \\
& =2 \sigma^{2} \operatorname{Re}\left\{\sum_{k=-(K+1)}^{K+1} \operatorname{Tr}\left[\mathbf{M}_{i} \mathbf{J}_{w}(-k) \mathbf{M}_{j}^{H} \mathbf{J}_{s}(k)\right]\right\}+O\left(\sigma^{4}\right)
\end{aligned}
$$

where $\mathbf{J}_{w}(k)=\left[\mathbf{J}_{w}(k)_{a b}\right]_{1 \leq a, b \leq r(K+1)}$ and $\mathbf{J}_{s}(k=$ $\left[\mathbf{J}_{s}(k)_{a b}\right]_{1 \leq a, b \leq K+L+1}$ are two shift matrices defined as

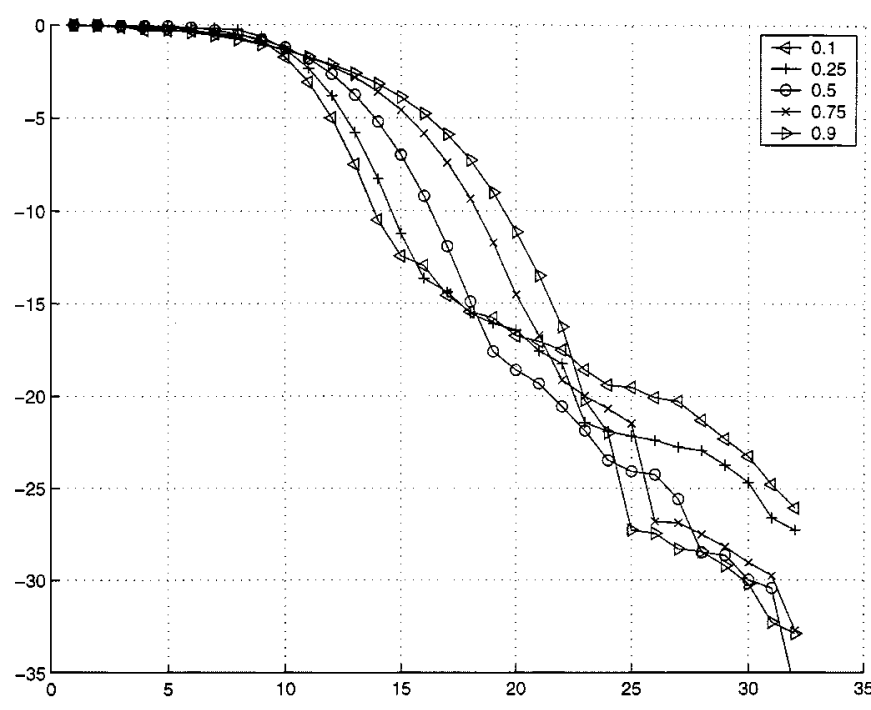

Fig. 3. Singular values of $\mathbf{G}_{L, d}(\boldsymbol{\tau})$ for various excess bandwidth factor $\alpha$, $p=2, B=3$.

$\mathbf{J}_{w}(k)_{a b}=\delta(a-b-r k)$ and $\mathbf{J}_{s}(k)_{a b}=\delta(a-b-k)$ and

$$
\begin{aligned}
\mathbf{M}_{i} & \triangleq \mathcal{T}_{K}\left(\mathbf{h}_{0}\right)^{H} \Gamma_{0} \mathbf{D}_{i} \mathbf{\Pi}_{0} \\
\Gamma_{0} & \triangleq\left(\mathbf{R}_{0}-\sigma^{2} \mathbf{I}\right)^{\#} \\
\mathbf{D}_{i} & \triangleq \sum_{j=1}^{d}\left[\frac{\partial^{2} \breve{J}}{\partial \tau^{2}}\left(\tau_{0}, \boldsymbol{\Pi}_{0}\right)^{-1}\right]_{i, j} \frac{\partial^{2} \breve{J}}{\partial \tau_{j} \partial \boldsymbol{\Pi}}\left(\tau_{0}, \boldsymbol{\Pi}_{0}\right)^{T}
\end{aligned}
$$

Proof: See Appendix C.

The expression of the covariance matrix involves the Hessian matrices of the criterion $\breve{J}(\tau, \Pi)$ at the true values of the delays and of the noise projector. Their calculations are made in [23]. We get

$$
\begin{aligned}
& \frac{\partial^{2} \breve{J}}{\partial \tau_{i} \partial \tau_{j}}\left(\tau_{0}, \boldsymbol{\Pi}_{0}\right) \\
& \quad=\frac{2 \operatorname{Re}\left\{\mathbf{a}_{0}^{H} \mathbf{G}_{\tau_{i}}^{\prime}\left(\boldsymbol{\tau}_{0}\right)^{H} \mathcal{D}\left(\boldsymbol{\Pi}_{0}\right) \mathbf{K} \mathcal{D}\left(\boldsymbol{\Pi}_{0}\right)^{H} \mathbf{G}_{\tau_{j}}^{\prime}\left(\boldsymbol{\tau}_{0}\right) \mathbf{a}_{0}\right\}}{\lambda_{\min }^{\alpha}\left(\mathbf{G}\left(\boldsymbol{\tau}_{0}\right)^{H} \mathbf{G}\left(\boldsymbol{\tau}_{0}\right)\right)\left\|\mathbf{a}_{0}\right\|^{2}}
\end{aligned}
$$

$$
\begin{aligned}
& \frac{\partial^{2} \breve{J}}{\partial \tau_{i} \partial \mathbf{\Pi}}\left(\boldsymbol{\tau}_{0}, \mathbf{\Pi}_{0}\right) \\
& \quad=\frac{\mathbf{U}^{H}+\mathbf{U}}{\lambda_{\min }^{\alpha}\left(\mathbf{G}\left(\boldsymbol{\tau}_{0}\right)^{H} \mathbf{G}\left(\boldsymbol{\tau}_{0}\right)\right)\left\|\mathbf{a}_{0}\right\|^{2}}
\end{aligned}
$$

where

$$
\operatorname{Vec}(\mathbf{U}) \triangleq\left(\mathbf{I}_{r(K+1)} \otimes \mathcal{T}_{K}\left(\mathbf{h}_{0}^{*}\right)\right) \mathbf{K} \mathcal{D}\left(\mathbf{\Pi}_{0}\right)^{H} \mathbf{G}_{\tau_{i}}^{\prime}\left(\boldsymbol{\tau}_{0}\right) \mathbf{a}_{0}
$$

with

$$
\begin{aligned}
\mathbf{K} \triangleq & \mathbf{I}_{r(K+1)\left(K+L_{0}+1\right)}-\mathcal{D}\left(\boldsymbol{\Pi}_{0}\right)^{H} \mathbf{G}\left(\boldsymbol{\tau}_{0}\right) \\
& \cdot\left(\mathbf{G}\left(\boldsymbol{\tau}_{0}\right)^{H} \mathcal{D}\left(\boldsymbol{\Pi}_{0}\right) \mathcal{D}\left(\mathbf{\Pi}_{0}\right)^{H} \mathbf{G}\left(\boldsymbol{\tau}_{0}\right)\right)^{\#} \\
& \cdot \mathbf{G}\left(\boldsymbol{\tau}_{0}\right)^{H} \mathcal{D}\left(\boldsymbol{\Pi}_{0}\right)
\end{aligned}
$$

and

$$
\left.\mathbf{G}_{\tau_{i}}^{\prime}\left(\tau_{0}\right) \triangleq \frac{\partial \mathbf{G}(\tau)}{\partial \tau_{i}}\right|_{\tau_{0}}
$$

1) Remarks: Note that the covariance of the estimator goes to zero as the noise variance tends to zero. This is related to the 
so-called "deterministic" estimation properties of the subspace algorithm; in absence of noise, the noise subspace can be estimated without errors from a finite number of samples, and the channel can be identified up to an arbitrary scalar factor (provided the identifiability conditions are satisfied).

We can see that the normalization factor $\lambda_{\min }^{\alpha}\left(\mathbf{G}\left(\boldsymbol{\tau}_{0}\right)^{H}\right.$ $\left.\mathbf{G}\left(\tau_{0}\right)\right)$ used to avoid spurious solutions does not influence the limiting variance of the estimates.

The asymptotic variance of the estimate otherwise depends in a rather intricate way on the emitting filter (and on the derivatives of the emitting filter), on the delays, and on the attenuations. To get a better understanding of the actual performance of the algorithm, we perform simulation in different scenarios.

\section{PERformance Evaluation}

In this section, the performance of the proposed parametric subspace method is assessed. An 8-PSK signal is modulated by a raised-cosine waveform with roll-off factor $\alpha=0.25$, truncated to a length of $L_{g}=4$ symbol periods. The signal is received on $q=2$ identical omnidirectional antennas, spaced by half a wavelength; standard far-field propagation conditions are assumed. The multipath propagation channel is characterized by a set of delays $\tau_{0}=\left(\tau_{01}, \cdots, \tau_{0 d_{0}}\right)$ and spatial signatures $\mathbf{a}_{0}=\left(\mathbf{a}_{01}, \cdots, \mathbf{a}_{0 d_{0}}\right)$. For the simulations, $\mathbf{a}_{0 k}=\beta_{0 k} \mathbf{a}\left(\theta_{0 k}\right)$, where $\beta_{0 k}$ and $\theta_{0 k}$ are, respectively, the $k$ th path attenuation factor and angle of incidence, and $\mathbf{a}(\theta)$ is the steering vector of a uniform linear array. We stress that this information is not exploited by the identification algorithm, which assumes arbitrary array geometry and propagation conditions. The oversampling factor is $p=2$ (compatible with the signal bandwidth). The signal is corrupted by an additive Gaussian noise. The SNR is defined as

$$
\mathrm{SNR}=\frac{\left\|\overrightarrow{\mathbf{h}}_{0}\right\|^{2}}{\sigma^{2}} \frac{1}{(1+\alpha) q}
$$

i.e., the average signal in the useful bandwidth divided by the number of sensors. The number of rays is always assumed to be known. The minimization of the cost function 19 with respect to $\tau$ is made using the iterative process described in Table I. To assess the robustness of the algorithm with respect to the channel order estimation error (which is known to be critical for the "plain" subspace method), the channel order is overestimated in all simulations. We set $\hat{L}=8$ (regardless of the "true" channel length, which is $\left.L_{g}+\left\lceil\left(\tau_{0 d}-\tau_{01}\right) / T\right\rceil\right)$. The size of the analysis window is $K=L+1$. The number of samples is set to $N=300$.

\section{A. Experimental Validation of the Asymptotic Performance Analysis}

We compare theoretical expressions obtained in Section V with empirical estimates obtained by Monte Carlo simulations. For each experiment, $T=1000$ Monte Carlo simulations are performed. We denote $C_{e}$ as the Monte Carlo mean-square estimation error $(\sqrt{N \tau-\mathrm{MSE}})$

$$
C_{e}=\sqrt{\frac{1}{T} \sum_{t=1}^{T} N\left\|\tau_{0}-\hat{\tau}\right\|^{2}} .
$$

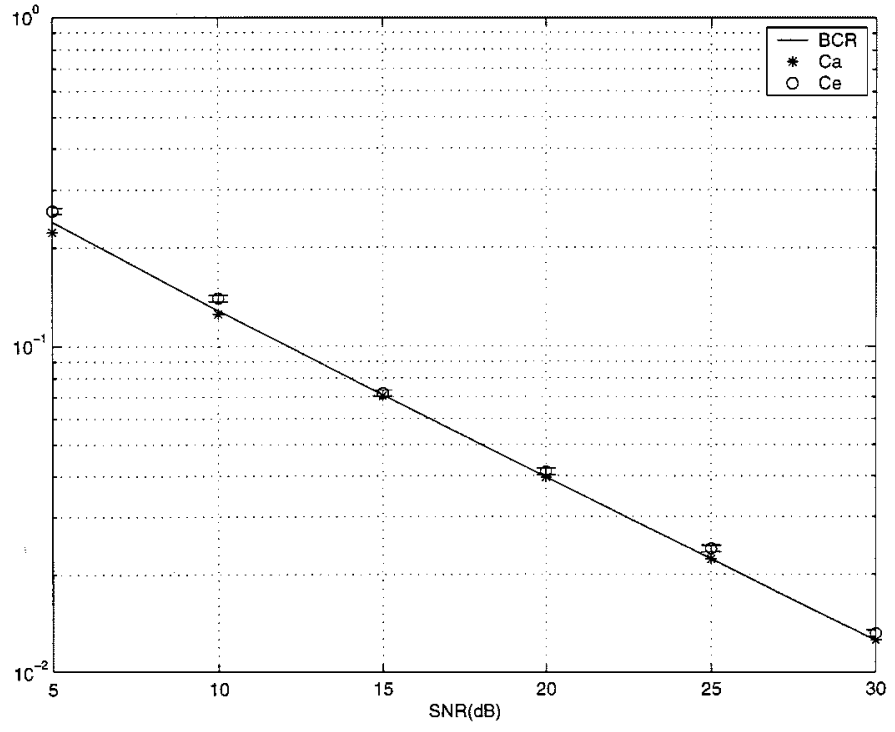

Fig. 4. Asymptotic mean-square error versus SNR for $\mathcal{M}_{0}^{(1)}$.

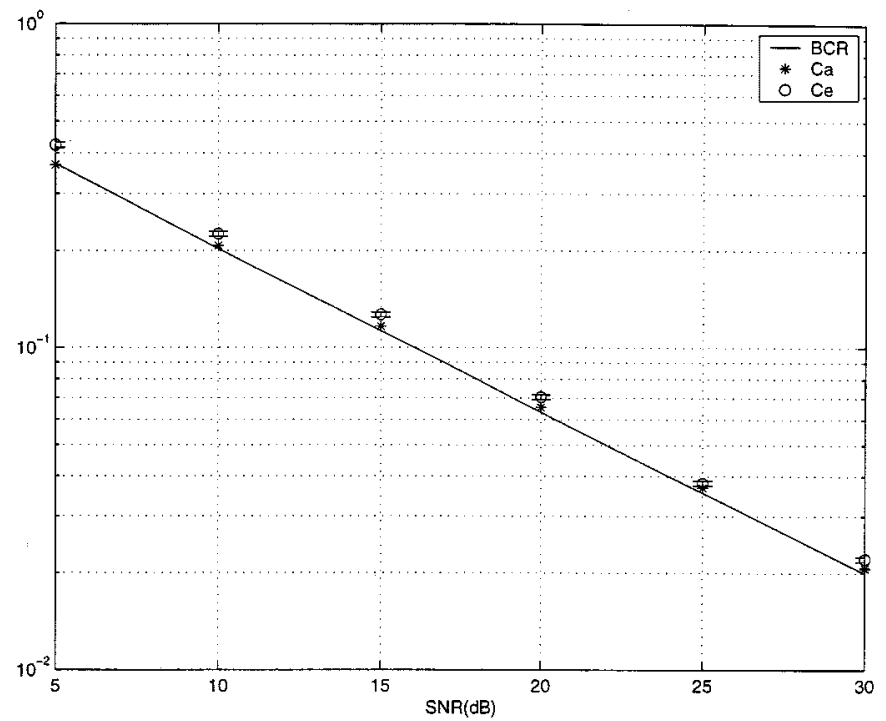

Fig. 5. Asymptotic mean-square error versus SNR for $\mathcal{M}_{0}^{(2)}$.

This quantity is compared with the square-root of the trace of the asymptotic covariance matrix $C_{a}=\sqrt{\operatorname{Tr}\left[\mathcal{C}\left(\tau, \sigma^{2}\right)\right]}$ and to the Gaussian Cramér-Rao lower bound (CRB), which provides a theoretical bound for all estimation procedures based on secondorder moments (see [1, App. C] and references therein). In these experiments, we use the following parameters:

$$
\begin{aligned}
\mathcal{M}_{0}^{(1)}= & \left\{d_{0}=1, \tau_{0}=0.2 T, \beta_{0}=1, \theta_{0}=0^{\circ}\right\} \\
\mathcal{M}_{0}^{(2)}= & \left\{d_{0}=2, \boldsymbol{\tau}_{0}=(0.22 .6) T, \boldsymbol{\beta}_{0}=(11), \boldsymbol{\theta}_{0}=(015)^{\circ}\right\} \\
\mathcal{M}_{0}^{(3)}= & \left\{d_{0}=3, \boldsymbol{\tau}_{0}=(0.22 .63 .4) T, \beta_{0}=(111)\right. \\
& \left.\boldsymbol{\theta}_{0}=(01530)^{\circ}\right\} .
\end{aligned}
$$

The SNR is varied between 0 and $30 \mathrm{~dB}$. Fig. 4 (respectively, Figs. 5 and 6) gives for $\mathcal{M}_{0}^{(1)}$ (respectively, $\mathcal{M}_{0}^{(2)}$ and $\mathcal{M}_{0}^{(3)}$ ) $C_{e}, C_{a}$, and the CRB as a function of the SNR. The error bars represent the standard deviation of $C_{e}$.

These figures demonstrate a close agreement between theoretical and experimental values, even for these reasonably small 


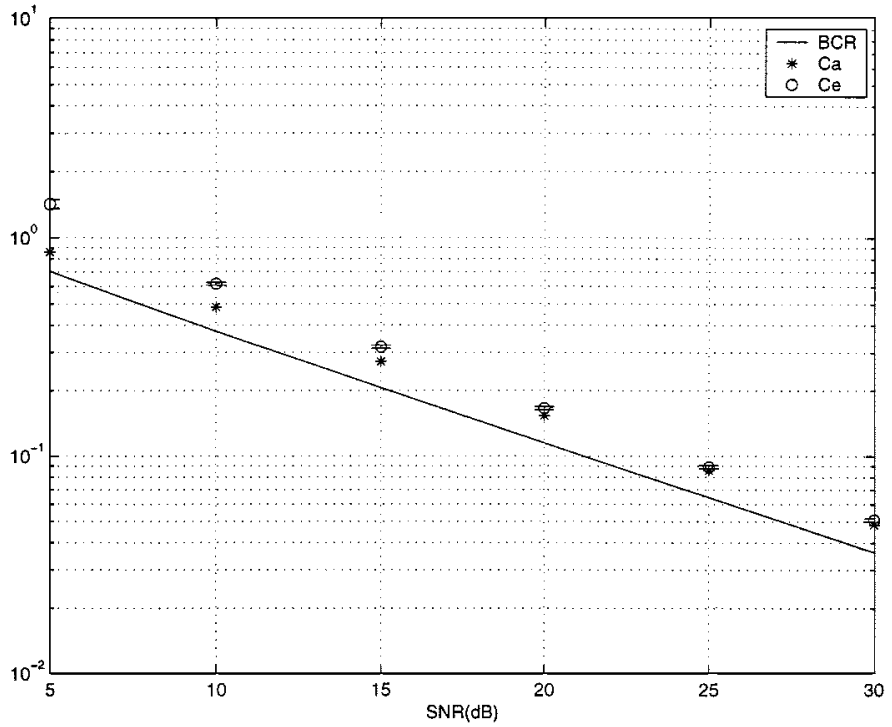

Fig. 6. Asymptotic mean-square error versus SNR for $\mathcal{M}_{0}^{(3)}$.

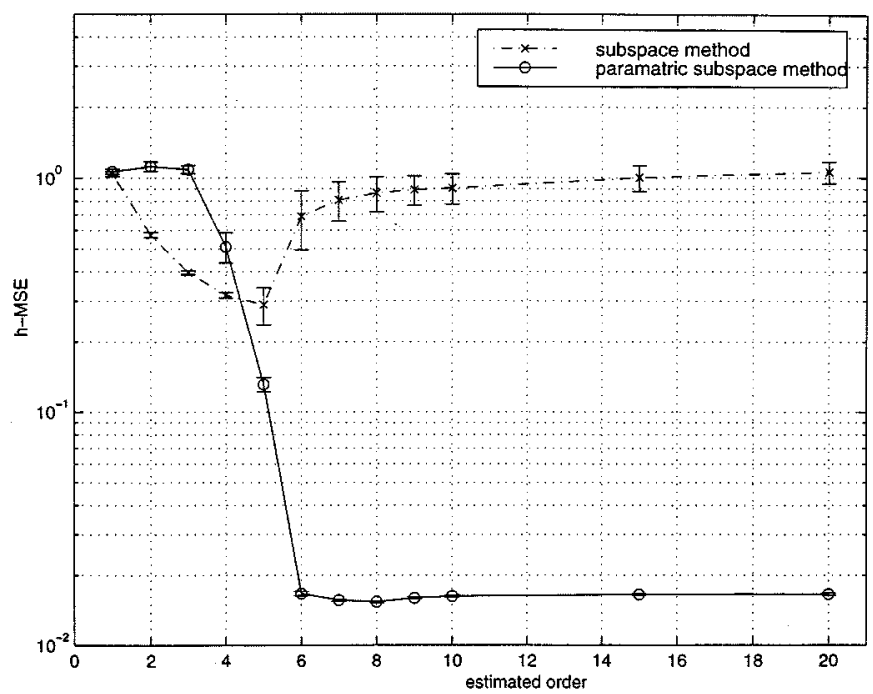

Fig. 7. Performance versus the estimated channel order $\hat{L}$.

sample sizes. It is worthwhile to note the close fit between the proposed estimator and the Gaussian CRB, showing that, at least for the first two scenarios considered (a single ray and two well-separated rays), our algorithm is almost second-order efficient.

\section{B. Numerical Study}

In this section, the SNR is set to $15 \mathrm{~dB}$. Fig. 7 gives the performance of proposed parametric subspace method and of the "plain" subspace method as a function of the assumed channel order $\hat{L}$ for the propagation channel $\mathcal{M}_{0}^{(2)}$. Comparison is given in terms of mean square error between the estimated channel $\hat{\overrightarrow{\mathbf{h}}}$ and the true channel $\overrightarrow{\mathbf{h}}_{0}$. The true value of the channel order is $L_{0}=L_{g}+\left\lceil\tau_{2}-\tau_{1}\right\rceil=7$. First, this simulation confirms the result of [20], which is recalled in Section III, i.e., performance of the "plain" subspace method is better for an underestimated value of the channel order. On the other hand, as $q=2 \geq d=2$, from Theorem 3, we know that the channel remains identifiable,

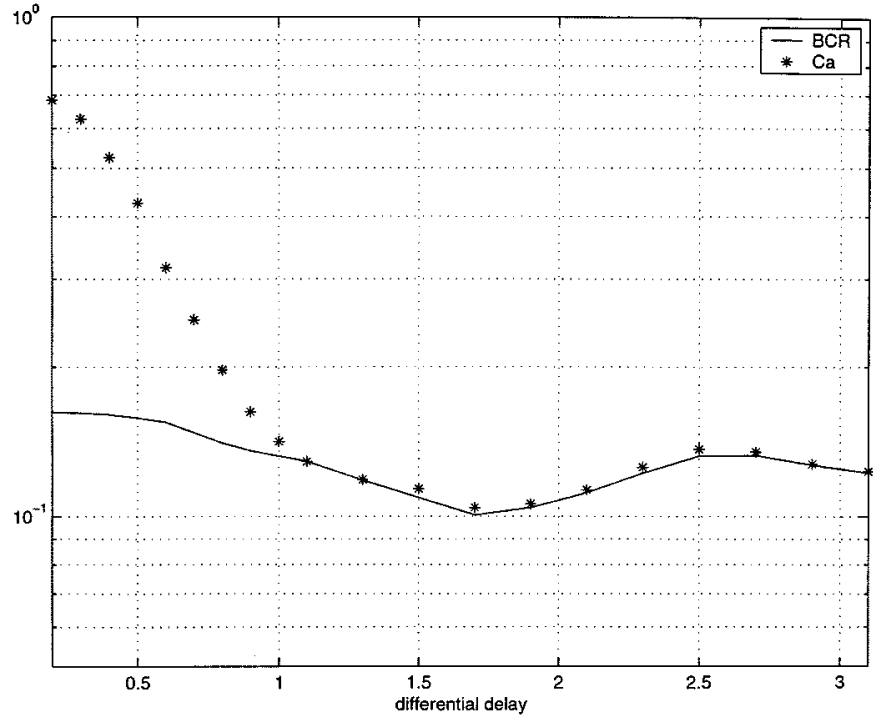

Fig. 8. Asymptotic mean-square error versus the differential delay.

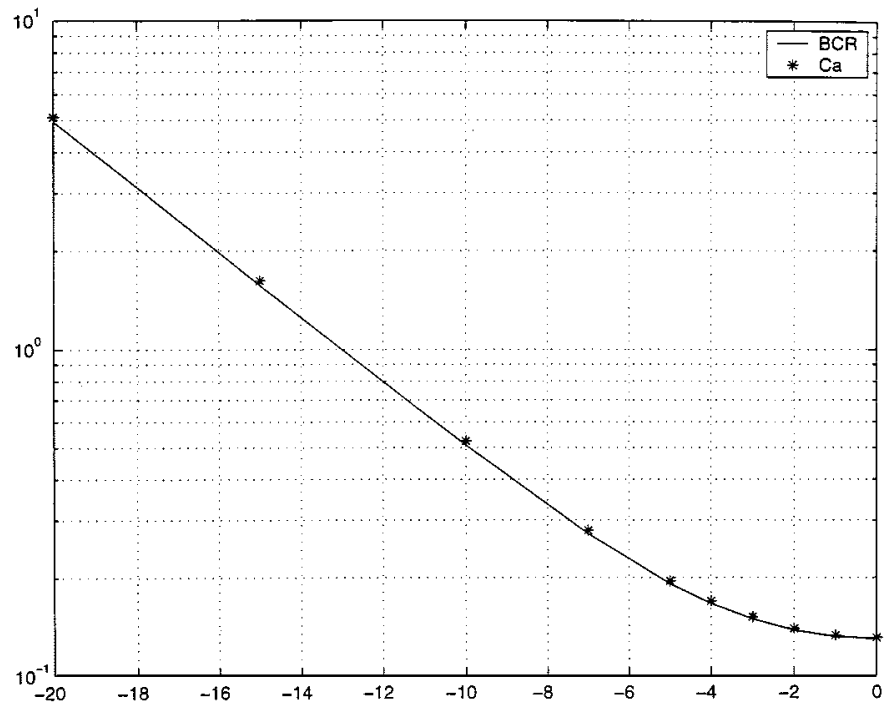

Fig. 9. Asymptotic mean-square error versus the gradient power.

whatever the order estimation. This point is asserted by this simulation. Note also that the performance is not significantly affected by the order overestimation, showing that the algorithm is robust to order overestimation.

Now, we investigate performance of the algorithm as a function of the propagation channel characteristics. The number of rays $d_{0}=2$, and $\theta_{0}=\left(\begin{array}{ll}0 & 15\end{array}\right)^{\circ}$. In Fig. 8, the two rays have the same power, and the differential delay is varied. In Fig. 9, the two delays are $\tau=\left[\begin{array}{ll}0.2 & 2.6\end{array}\right] T$, and the power gradient between the two rays is varied: $10 \log _{10}\left(\beta_{2} / \beta_{1}\right)$. Fig. 8 shows that performance of the proposed criterion are affected when the differential delay between the two rays becomes small.

\section{IMPROVEMENTS OF THE METHOD FOR BLIND EQUALIZATION}

\section{A. Generalized Algorithm}

The previous section showed that performance is affected by small differential delay. We suggest here a new algorithm that 
avoids this problem when the parameter of interest is the global channel response $\underline{\mathbf{h}}_{0}(z)$ (and not the value of the differential delay). Using the principle proposed in [3] for direction-of-arrival estimation, we consider a generalized model of the propagation consisting for closed delays in a linear combination of $g(\tau)$ and of its gradient w.r.t. to $\tau$. Denote $c$ as the number of delays that would be too close to be estimated separately $c \leq\lfloor d / 2\rfloor$. Then, there exists a permutation function $\sigma$ such that

$$
\begin{aligned}
h(t)= & \sum_{k=1}^{c} \mathbf{a}_{\sigma(k)} g\left(t-\tau_{\sigma(k)}\right)+\mathbf{a}_{\sigma(d-c+k)} g\left(t-\tau_{\sigma(k)}+\delta \tau_{\sigma(k)}\right) \\
& +\sum_{k=c+1}^{d-c} \mathbf{a}_{\sigma(k)} g\left(t-\tau_{\sigma(k)}\right)
\end{aligned}
$$

Define the gradient $f(\tau)=\partial g / \partial \tau$. Then, eventually, with some modifications on the index of the rays, but without loss of generality, the first Taylor-order expansion of $\mathbf{h}(t)$ yields

$$
\mathbf{h}(t) \simeq \sum_{k=1}^{c}\left(\mathbf{u}_{k} g\left(t-\tau_{k}\right)+\mathbf{v}_{k} f\left(t-\tau_{k}\right)\right)+\sum_{k=c+1}^{d-c} \mathbf{a}_{k} g\left(\tau_{k}\right)
$$

where $\mathbf{u}_{k}$ and $\mathbf{v}_{k}$ are $q$ vectors. Hence, proceeding as in Section IV and using the same notations, we get

$$
\overrightarrow{\mathbf{h}} \simeq\left[\begin{array}{lll}
\mathbf{G}_{L, c}(\tau) & \mathbf{F}_{L, c}(\tau) & \mathbf{G}_{L, d-2 c}(\tau)
\end{array}\right]\left[\begin{array}{lll}
\mathbf{u}^{T} & \mathbf{v}^{T} & \mathbf{a}^{T}
\end{array}\right]^{T} .
$$

Letting $\hat{L}, \hat{d}$, and $\hat{c}$ be the estimated values, we define the following generalized criterion: $\dot{J}_{\hat{L}, \hat{d}, \hat{c}}\left(\left[\tau_{1}, \cdots, \tau_{\hat{d}-\hat{c}}\right]\right)=$ $\lambda_{\min }\left(\mathbf{P}_{\hat{L}, \hat{d}, \hat{c}}(\boldsymbol{\tau}, \hat{\mathbf{R}})\right)$, where

$$
\begin{aligned}
\mathbf{P}_{L, d, c}(\boldsymbol{\tau}, \mathbf{R})= & {\left[\begin{array}{c}
\mathbf{G}_{L, c}(\boldsymbol{\tau})^{H} \\
\mathbf{F}_{L, c}(\boldsymbol{\tau})^{H} \\
\mathbf{G}_{L, d-2 c}(\boldsymbol{\tau})^{H}
\end{array}\right] \mathbf{Q}_{L}(\mathbf{R}) } \\
& \cdot\left[\begin{array}{lll}
\mathbf{G}_{L, c}(\boldsymbol{\tau}) & \mathbf{F}_{L, c}(\boldsymbol{\tau}) & \mathbf{G}_{L, d-2 c}(\boldsymbol{\tau})
\end{array}\right] .
\end{aligned}
$$

\section{B. Joint Number of Delays and Channel Response Estimation}

The previous algorithm requires estimates of the number of delays and estimates of the number of couples of delays. We suggest here the selection of these parameters using a criterion based on the equalized signals. More precisely, for a given estimate of $\mathbf{h}$ (associated with a given number of delays $d$ and of couples $c$ ), we compute an equalized signal $\hat{s}(k)=\hat{s}(\mathbf{h}, k)$, using e.g., an MSE equalizer. Then, we defined a criterion based on these equalized signals. Because the modulation here is of constant modulus, it is appropriate to use a criterion assessing the distance between the modulus equalized signal and the nominal value of this module

$$
\Omega(\hat{\overrightarrow{\mathbf{h}}})=\frac{1}{N} \sum_{k=1}^{N}(|\hat{s}(k)|-1)^{2}
$$

This criterion is used in the CMA algorithm for blind identification. Then, the joint algorithm may be summarized as follows:
- For all values of $d$ between 1 and $d_{\max }$ and for all values of $c$ between 1 and $\lfloor\hat{d} / 2\rfloor$, do the following.

- Estimate $\hat{\boldsymbol{\tau}}=\arg \min _{\boldsymbol{\tau}} \dot{J}_{\hat{L}, \hat{d}, \hat{c}}(\boldsymbol{\tau}) \quad$ and $\left[\hat{\mathbf{u}}^{T} \hat{\mathbf{v}}^{T} \hat{\mathbf{a}}^{T}\right]^{T}=\operatorname{vp}_{\min }\left(\mathbf{P}_{\hat{L}, \hat{d}, \hat{c}}(\hat{\tau}, \hat{\mathbf{R}})\right)$.

- Obtain an estimate of the channel response $\hat{\overrightarrow{\mathbf{h}}}$ using (34).

- Calculate the value of $\Omega(\hat{\overrightarrow{\mathbf{h}}}, d, c)$.

- $(\hat{\overrightarrow{\mathbf{h}}}, \hat{d}, \hat{c})=\arg \min \Omega(\overrightarrow{\mathbf{h}}, d, c)$.

At each step, the delays are estimated by a monodimensional search using the previously obtained delays as described in Step 4 of Table I.

\section{Random Test Evaluation}

The performance of the algorithm is assessed by the following random test evaluation. $T=1000$ bursts of $N=256$ eight-PSK symbols are simulated. For each burst, the number of rays and the delays are chosen randomly within limits $1 \leq d_{0} \leq 4$, $0 \leq \tau_{0 k} \leq 10 T$. The angle of incidence are fixed, and $\theta_{k}=$ $(k-1) \cdot 10^{\circ}$ for $1 \leq k \leq d_{0}$. To make the experiment more realistic, we assume that the power of the rays $|\beta|$ decreases linearly with the delay in such a way that $|\beta(\tau=0)|=1$, and $|\beta(\tau=10 T)|=0.1$. The phase of the complex attenuation factor $\beta$ is also random. These values are typical of the STANAG 4285 modems used for long-distance communication over the ionospheric channel at $2400 \mathrm{~b} / \mathrm{s}$. The signal is received on $q=3$ antennas. The pulse shape is truncated at a length of $L_{g}=10$ symbol periods. The channel order is set at a value compatible with the maximum delay spread $\hat{L}=20 \geq L_{g}+10$. The proposed blind equalization algorithm is compared with the SIMO block-constant modulus algorithm (CMA) [14]. A simulation result is given in symbol error rate as a function of the SNR. The proposed algorithm significantly outperforms the CMA (see Fig. 10).

\section{CONCLUSION}

In this paper, assuming the knowledge of the pulse-shape filter, a parametric subspace method has been presented for the blind identification of specular propagation channels. Contrary to the classical subspace method, the parametric method exploits the specular structure of the propagation channel, which makes it very robust to channel order overestimation. By the reduction and the normalization of the parametric subspace criterion, we have obtained a simpler algorithm that does not suffer from "spurious" global minima. Identifiability conditions have been given and show that the estimates are consistent in most situations of practical interest. The analytical performance analysis, which was confirmed by limited Monte-Carlo simulations, shows the asymptotic behavior of the method compare with the Gaussian CRB.

\section{APPENDIX A PROOF OF THEOREM 1}

The proof given here uses the theory of the rational subspace. This theory is not recalled here, but we will use vocabulary and notations as presented in [1]. 


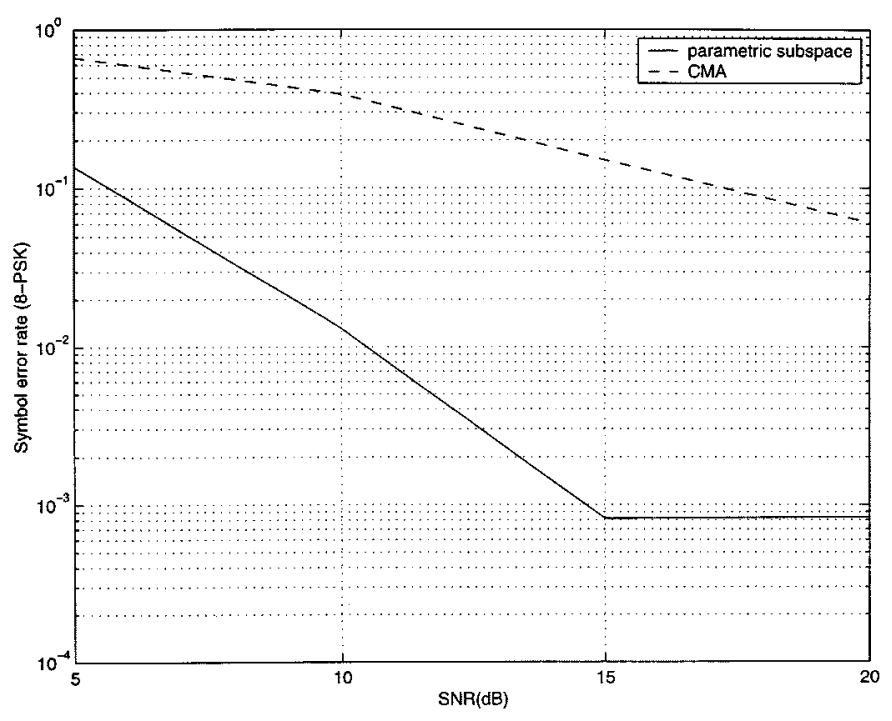

Fig. 10. Performance of proposed equalization algorithm and CMA.

Denote $\mathcal{E}\left(\mathbf{h}_{0}\right)$ as the rational subspace spanned by $\underline{\mathbf{h}}_{0}(z)$ and $\mathcal{E}\left(\mathbf{h}_{0}\right)^{\perp}$ as the dual space of $\mathcal{E}\left(\mathbf{h}_{0}\right)$. Let $\left[\underline{\mathbf{g}}_{1}(z), \cdots, \underline{\mathbf{g}}_{r-1}(z)\right]$ be a minimal polynomial basis of $\mathcal{E}\left(\mathbf{h}_{0}\right)^{\perp}$ with indices $L_{1}^{\perp} \leq \cdots \leq L_{r-1}^{\perp}$, where $L_{i}^{\perp}=\operatorname{degg}_{i}(z)$. This implies (see Forney [8]) that every polynomial vector $\underline{\mathbf{g}}(z)$ in $\mathcal{E}\left(\mathbf{h}_{0}\right)^{\perp}$ writes $\underline{\mathbf{g}}(z)=\sum_{i=1}^{r-1} r_{i}(z) \underline{\mathbf{g}}_{i}(z)$, where $r_{i}(z)$ are scalar polynomials, and $\operatorname{deg} \underline{g}(z)=\max _{1 \leq i \leq r-1}\left(\operatorname{deg} r_{i}(z)+L_{i}^{\perp}\right)$. Using [8, th. 3] (see also [1] and [5]), we have $L_{0}=\sum_{i=1}^{r-1} L_{i}^{\perp}$; thus since, under the stated assumptions $K \geq \hat{L} \geq L_{0}$, we have $K \geq L_{r-1}^{\perp} \geq \cdots \geq L_{1}^{\perp}$. Denote $\mathbf{G}_{i}(z)$ as the polynomial matrix of size $r \times\left(K-L_{i}^{\perp}+1\right)$ defined by the $K-L_{i}^{\perp}+1$ "shifted" versions of $\underline{\mathbf{g}}_{i}(z), \mathbf{G}_{i}(z)=\left[\underline{\mathbf{g}}_{i}(z) z^{-1} \underline{\mathbf{g}}_{i}(z) \cdots z^{-K+L_{i}^{\perp}} \underline{\mathbf{g}}_{i}(z)\right]$. $\mathbf{G}(z)=\left[\mathbf{G}_{1}(z) \cdots \mathbf{G}_{r-1}(z)\right]$ forms a basis over $\mathbb{C}$ of $\mathcal{P}_{K}\left(\mathcal{E}\left(\mathbf{h}_{0}\right)^{\perp}\right)$, where $\mathcal{P}_{K}\left(\mathcal{E}\left(\mathbf{h}_{0}\right)^{\perp}\right)$ denotes the linear subspace over $\mathbb{C}$ of polynomial vectors of degrees less than or equal to $K$ belonging to $\mathcal{E}\left(\mathbf{h}_{0}\right)^{\perp}$.

It has been shown in [1] (Theorem 4) that $\mathcal{P}_{K}\left(\mathcal{E}\left(\mathbf{h}_{0}\right)^{\perp}\right)$ is isomorphic to $\operatorname{Null}\left(\mathcal{T}_{K}\left(\mathbf{h}_{0}\right)^{H}\right)$, i.e., every polynomial vector $\mathcal{P}_{K}\left(\mathcal{E}\left(\mathbf{h}_{0}\right)^{\perp}\right)$ is associated with a single vector $\overrightarrow{\mathbf{g}}$ in $\operatorname{Null}\left(\mathcal{T}_{K}\left(\mathbf{h}_{0}\right)^{H}\right)$. This last result means that the set of vectors $\left\{\overrightarrow{\mathbf{g}}_{i}^{j}\right\} \quad 1 \leq i \leq r-1,0 \leq j \leq K-L_{i}^{\perp}$ associated with $z^{-j} \mathbf{g}_{i}(z)$ and, thus, defined as $\overrightarrow{\mathbf{g}}_{i}^{j}=$ $[\underbrace{0 \cdots 0} \underline{\mathrm{g}}_{i}(0)^{T} \cdots \underline{\mathrm{g}}_{i}\left(L_{i}^{\perp}\right)^{T} 0 \cdots 0]_{r(K+1) \times 1}^{T}$ forms a basis of $\stackrel{j r}{\operatorname{Span}}\left(\boldsymbol{\Pi}_{L_{0}}\right)$.

Now, we show by contradiction that for all $1 \leq i \leq r-1$, there exists a scalar polynomial $r_{i}(z), \operatorname{deg} r_{i}(z) \leq \bar{K}-L_{i}^{\perp}$ such that the vector $\overrightarrow{\mathbf{f}}_{i}$, associated with $\underline{\mathbf{f}}_{i}(z)=r_{i}(z) \underline{\mathbf{g}}_{i}(z)$, belongs to $\operatorname{Span}\left(\boldsymbol{\Pi}_{\hat{L}}\right)$. Assume that this is false for some $i$; then, $\operatorname{Span}\left(\left\{\overrightarrow{\mathbf{g}}_{i}^{j}\right\}_{j}\right) \cap \operatorname{Span}\left(\boldsymbol{\Pi}_{\hat{L}}\right)=\varnothing$ and $\operatorname{Rank}\left(\boldsymbol{\Pi}_{\hat{L}}\right) \leq \operatorname{Rank}\left(\boldsymbol{\Pi}_{L_{0}}\right)-\operatorname{dim}\left(\operatorname{Span}\left(\left\{\overrightarrow{\mathbf{g}}_{i}^{j}\right\}_{j}\right)\right)$. As $\operatorname{dim}\left(\operatorname{Span}\left(\left\{\overrightarrow{\mathrm{g}}_{i}^{j}\right\}_{j}\right)\right)=K-L_{i}^{\perp}+1$ and from $\operatorname{Rank}\left(\boldsymbol{\Pi}_{L_{0}}\right)=r(K+1)-\left(K+L_{0}+1\right)$, we get

$$
\begin{aligned}
\operatorname{Rank}\left(\boldsymbol{\Pi}_{\hat{L}}\right) & \leq r(K+1)-\left(K+L_{0}+1\right)-\left(K-L_{i}^{\perp}+1\right) \\
& \leq(r-1)(K+1)-(K+1) .
\end{aligned}
$$

Now, by assumption $\operatorname{Rank}\left(\Pi_{\hat{L}}\right)=(r-1)(K+1)-\hat{L}>(r-$ 1) $(K+1)-(K+1)$ as $K \geq \hat{L}$. This results in a contradiction. Thus, for all $1 \leq i \leq r-1$, there exists a polynomial $r_{i}(z)$, $\operatorname{deg} r_{i}(z) \leq K-L_{i}^{\perp}$ such that the vector $\overrightarrow{\mathrm{f}}_{i}$ associated with $\underline{\mathbf{f}}_{i}(z)=r_{i}(z) \underline{\mathbf{g}}_{i}(z)$ verifies

$$
\overrightarrow{\mathbf{f}}_{i} \mathcal{T}_{K}(\hat{\mathbf{h}})=0 \Leftrightarrow\left(r_{i}(z) \underline{\mathbf{g}}_{i}(z)\right)^{T} \underline{\hat{\mathbf{h}}}(z)=0 \Leftrightarrow \underline{\mathbf{g}}_{i}(z)^{T} \underline{\hat{\mathbf{h}}}(z)=0 .
$$

Thus, $\underline{\hat{\mathbf{h}}}(z)$ belongs to the dual space of $\mathcal{E}\left(\mathbf{h}_{0}\right)^{\perp}$, i.e., $\mathcal{E}\left(\mathbf{h}_{0}\right)$. Hence, by definition of $\mathcal{E}\left(\mathbf{h}_{0}\right), \underline{\hat{\mathbf{h}}}(z)=c(z) \underline{\mathbf{h}}_{0}(z)$.

\section{APPENDIX B \\ PROOF OF THEOREM 2}

First, let us introduce some notations. If $\mathcal{D}=$ $\left\{\tau_{1}, \tau_{2}, \cdots, \tau_{d}\right\}$, then we denote $\mathcal{D}^{n}=\left\{\tau_{1}+n T, \tau_{2}+\right.$ $\left.n T, \cdots, \tau_{d}+n T\right\}$. Let denote $\{\tau\}^{M}=\{\tau, \tau+T, \cdots, \tau+$ $M T\}$ and use $\mu=\min (q, d)$.

The polynomial $c(z)$ not being identically equal to zero, there exists $m \in\{0, \cdots, M\}$ such that $c_{m} \neq 0$. Let $\mathcal{D}_{0}=\left\{\tau_{01}, \tau_{02}, \cdots, \tau_{0 d_{0}}\right\}$ and $\hat{\mathcal{D}}=\left\{\hat{\tau}_{1}, \hat{\tau}_{2}, \cdots, \hat{\tau}_{\hat{d}}\right\}$ be, respectively, the set of the true and of the estimated delays. In a first step, we will show that for any $k\left(1 \leq k \leq d_{0}\right)$ $\tau_{0 k}+m T \in \hat{\mathcal{D}}$, i.e., $\mathcal{D}_{0}^{m} \subset \hat{\mathcal{D}}$.

Under I1, it holds that $\operatorname{card}\left(\mathcal{C}_{k}\left(\mathcal{M}_{0}\right)\right) \leq \min (q, d)=\mu$, and thus, $\operatorname{card}\left(\mathcal{D}_{0} \backslash \mathcal{C}_{k}\left(\mathcal{M}_{0}\right)\right) \geq d_{0}-\mu$, and we can extract a subset $\mathcal{T}_{k} \subset \mathcal{D}_{0} \backslash \mathcal{C}_{k}\left(\mathcal{M}_{0}\right)$ such that $\operatorname{card}\left(\mathcal{T}_{k}\right)=\left(d_{0}-\mu\right)$. Then, under I2, we may define an oblique projector $\mathbf{P}_{k}$ verifying

$$
\left\{\begin{array}{l}
\mathbf{P}_{k} \mathbf{a}_{0 k}=\mathbf{a}_{0 k} \\
\mathbf{P}_{k} \mathbf{a}_{0 i}=0
\end{array} \forall i, \tau_{0 i} \in \mathcal{D}_{0} \backslash\left(\mathcal{T}_{k} \cup\left\{\tau_{0 k}\right\}\right) .\right.
$$

We introduce following notations: $\mathbf{P}_{k} \mathbf{a}_{0 i}=v_{k, i} \mathbf{a}_{0 k}$ for all $\tau_{0 i} \in \mathcal{T}_{k}$ and $\mathbf{P}_{k} \hat{\mathbf{a}}_{i}=\hat{v}_{k, i} \mathbf{a}_{0 k}$ for all $1 \leq i \leq \hat{d}$.

Noting that $g_{T / p}(\tau ; z) z^{-s p}=\overline{g_{T / p}}(\tau+s T ; z)$ and $c\left(z^{p}\right) g_{T / p}(\tau ; z)=\sum_{s=0}^{M} c_{s} g_{T / p}(\tau+s T ; z)$, the equation $\hat{\mathbf{h}}_{T / p}(z)=c\left(z^{p}\right) \mathbf{h}_{0(T / p)}(z)$ may be rewritten as

$$
\sum_{k=1}^{\hat{d}} \hat{\mathbf{a}}_{k} g_{T / p}\left(\hat{\tau}_{k} ; z\right)=\sum_{s=0}^{M} \sum_{k=1}^{d_{0}} c_{s} \mathbf{a}_{0 k} g_{T / p}\left(\tau_{0 k}+s T ; z\right) .
$$

Applying $\mathbf{P}_{k}$ to (35) yields

$$
\begin{aligned}
\sum_{\hat{\tau}_{i} \in \hat{\mathcal{D}}} \hat{v}_{k, i} g_{T / p}\left(\hat{\tau}_{i} ; z\right) & \\
= & \sum_{s=0}^{M} c_{s} g_{T / p}\left(\tau_{0 k}+s T ; z\right) \\
& +\sum_{s=0}^{M} \sum_{\tau_{0 i} \in \mathcal{T}_{k}} c_{s} v_{k, i} g_{T / p}\left(\tau_{0 i}+s T ; z\right) .
\end{aligned}
$$

Define $\mathcal{S}_{k}=\bigcup_{\tau_{0 i} \in\left\{\tau_{0 k} \cup \mathcal{T}_{k}\right\}}\left\{\tau_{0 i}\right\}^{M}$. The set $\left(\mathcal{S}_{k} \cup \hat{\mathcal{D}}\right)$ contains all the delays appearing in (36). We will show that under I3, the set of delays $\left(\mathcal{S}_{k} \cup \hat{\mathcal{D}}\right)$ verifies the condition given by (20). From the structure of $\mathcal{S}_{k}$ [using $\operatorname{card}\left(\mathcal{T}_{k} \cup \tau_{0 k}\right)=d_{0}-\mu+1$ ], we deduce that

$$
\begin{aligned}
& \operatorname{card}\left(\mathcal{S}_{k}\right) \leq(M+1)\left(d_{0}-\mu+1\right) \\
& \operatorname{card}\left(\mathcal { S } _ { k } \cap \left[n T / p, n T / p+T[) \leq d_{0}-\mu+1, \quad \forall n \in \mathbb{N} .\right.\right.
\end{aligned}
$$


Hence, for all $(n, l) \in \mathbb{N}^{2}$, it holds that

$$
\begin{aligned}
\operatorname{card} & \left(\mathcal{S}_{k} \cap[n T / p,(n+l+1) T / p[)\right. \\
& \leq \operatorname{card}\left(\mathcal{S}_{k} \cap[n T / p, n T / p+\lceil(l+1) / p\rceil T]\right) \\
& \leq \min (M+1,\lceil(l+1) / p\rceil)\left(d_{0}-\mu+1\right) .
\end{aligned}
$$

Defining the function of $l, \eta(l)=p L_{g}+l-\min (M+1,\lceil(l+$ 1) $/ p\rceil)\left(d_{0}-\mu+1\right)-\hat{d}$, one can see that

$$
\text { if } d_{0}-\mu-p+1>0 \arg \min _{l} \eta(l)=M p
$$

and

$$
\begin{gathered}
\eta(M p)=p L_{g}-\hat{d}-\left(d_{0}-\mu+1\right)+M\left(d_{0}-\mu-p+1\right) \\
\quad \text { if } d_{0}-\mu-p+1 \leq 0 \arg \min _{l} \eta(l)=0
\end{gathered}
$$

and

$$
\eta(0)=p L_{g}-\hat{d}-\left(d_{0}-\mu+1\right) .
$$

Thus, for all $l \in \mathbb{N}, \eta(l) \geq p L_{g}-\hat{d}-\left(d_{0}-\mu+1\right)-\max \left(0, d_{0}-\right.$ $\mu-p+1) M$. Hence, under I3, for all $l \in \mathbb{N}, \eta(l) \geq 0$, and thus, we obtain the required result, i.e., for all $(n, l) \in \mathbb{N}^{2}$

$$
\operatorname{card}\left(( \mathcal { S } _ { k } \cup \hat { \mathcal { D } } ) \cap \left[n T / p,(n+l+1) T / p[) \leq p L_{g}+l .\right.\right.
$$

The set of distinct delays of $\left(\mathcal{S}_{k} \cup \hat{\mathcal{D}}\right)$ satisfies the conditions of the property $\mathrm{P} 1$. Thus, if a delay $\tau$ appears only one time in the set $\left(\mathcal{S}_{k} \cup \hat{\mathcal{D}}\right)$, then from $\mathrm{P} 1$, the coefficient affecting $g(\tau ; z)$ in (36) is equal to zero. Thus, $c_{m} \neq 0$ implies that $\tau_{0 k}+m T \in$ $\left.\left(\mathcal{S}_{k} \cup \hat{\mathcal{D}}\right) \backslash\left\{\tau_{0 k}+m T\right\}\right)$. However, as $\mathcal{T}_{k}$ has been constructed in such a way that $\tau_{0 k}+m T \notin \mathcal{S}_{k}$, then $\tau_{0 k}+m T \in \hat{\mathcal{D}}$. Repeating the same argument for $1 \leq k \leq d_{0}$ shows that $\mathcal{D}_{0}^{m} \subset \hat{\mathcal{D}}$. We can assume that $\hat{\tau}_{k}=\tau_{0 k}+m T$ for all $1 \leq k \leq d_{0}$.

Now, assume that $m^{\prime} \neq m$ exists such that $c_{m^{\prime}} \neq 0$. Proceeding as above, it may be shown that $\mathcal{D}_{0}^{m^{\prime}} \subset \hat{\mathcal{D}}$, and thus, $\left(\mathcal{D}_{0}^{m} \cup \mathcal{D}_{0}^{m^{\prime}}\right) \subset \hat{\mathcal{D}}$. However

$$
\begin{aligned}
& \operatorname{card}\left(\mathcal{D}_{0}^{m} \cup \mathcal{D}_{0}^{m^{\prime}}\right) \\
& \quad=2 d_{0}-\operatorname{card}\left\{\left(\tau_{0 i}, \tau_{j}\right) \in \mathcal{D}_{0} ; \tau_{0 i}+m T=\tau_{j}+m^{\prime} T\right\} \\
& \quad \leq 2 d_{0}-\max _{n \in \mathbb{Z}^{*}} \operatorname{card}\left\{\left(\tau_{0 i}, \tau_{j}\right) \in \mathcal{D}_{0} ; \tau_{0 i}=\tau_{j}+n T\right\}
\end{aligned}
$$

Thus, under I4, $\operatorname{card}\left(\mathcal{D}_{0}^{m} \cup \mathcal{D}_{0}^{m^{\prime}}\right) \geq 2 d_{0}-\delta>\operatorname{card}(\hat{\mathcal{D}})$, which results in a contradiction, and $c(z)=c_{m} z^{-m}$.

Finally, plugging the previous result into (35) yields

$$
\sum_{k=1}^{d_{0}}\left(c_{m} \mathbf{a}_{0 k}-\hat{\mathbf{a}}_{k}\right) g_{T / p}\left(\hat{\tau}_{k} ; z\right)-\sum_{k=d_{0}+1}^{\hat{d}} \hat{\mathbf{a}}_{k} g_{T / p}\left(\hat{\tau}_{k} ; z\right)=0
$$

As $p L_{g} \geq \hat{d}$, from property $\mathrm{P} 1,\left\{g\left(\hat{\tau}_{k} ; z\right)\right\}$ are independent. Hence, $\mathbf{a}_{0 k} c_{m}-\hat{\mathbf{a}}_{k}=0$ for $1 \leq k \leq d_{0}$, and $\hat{\mathbf{a}}_{k}=0$ for $d_{0}+1 \leq k \leq \hat{d}$.

\section{APPENDIX C}

The estimator of the delays are obtained by solving the equation $(\partial \breve{J} / \partial \tau)(\hat{\boldsymbol{\tau}}, \hat{\mathbf{\Pi}})=0$. By expanding this relation around $\left(\tau_{0}, \Pi_{0}\right)$, the true value of the delays, and of the noise projector, we may write

$$
\begin{aligned}
& \frac{\partial \breve{J}}{\partial \tau}(\hat{\tau}, \hat{\Pi}) \\
& \quad=\frac{\partial \breve{J}}{\partial \tau}\left(\tau_{0}, \hat{\Pi}\right)+\frac{\partial^{2} \breve{J}}{\partial \tau^{2}}\left(\tau_{0}, \hat{\Pi}\right)\left(\hat{\tau}-\tau_{0}\right)+o\left(\hat{\tau}-\tau_{0}\right)
\end{aligned}
$$

where $(\partial \breve{J} / \partial \boldsymbol{\tau})(\boldsymbol{\tau}, \boldsymbol{\Pi})$ denote the gradient of $\breve{J}(\boldsymbol{\tau}, \boldsymbol{\Pi})$ and $\left(\partial^{2} J / \partial \tau^{2}\right)(\tau, \Pi)$ the Hessian matrix. Note that $\left(\partial \breve{J} / \partial \tau_{i}\right)\left(\tau_{0}, \Pi_{0}\right)=0$, and thus, the Jacobian $(\partial \breve{J} / \partial \boldsymbol{\tau})\left(\boldsymbol{\tau}_{0}, \hat{\boldsymbol{\Pi}}\right)$ may be expressed as

$$
\begin{aligned}
& {\left[\frac{\partial \breve{J}}{\partial \tau}\left(\tau_{0}, \hat{\mathbf{\Pi}}\right)\right]_{i}} \\
& \quad=\frac{\partial \breve{J}}{\partial \tau_{i}}\left(\tau_{0}, \hat{\Pi}\right) \\
& \quad=\operatorname{Tr}\left[\frac{\partial^{2} \breve{J}}{\partial \tau_{i} \partial \boldsymbol{\Pi}}\left(\tau_{0}, \Pi_{0}\right)^{T}\left(\hat{\Pi}-\Pi_{0}\right)\right]+o\left(\hat{\Pi}-\Pi_{0}\right) .
\end{aligned}
$$

On the other hand, standard results on the perturbation of the eigenprojectors (see [17]) show that

$$
\hat{\boldsymbol{\Pi}}-\boldsymbol{\Pi}_{0}=-\Gamma_{0}\left(\hat{\mathbf{R}}-\mathbf{R}_{0}\right) \boldsymbol{\Pi}_{0}-\boldsymbol{\Pi}_{0}\left(\hat{\mathbf{R}}-\mathbf{R}_{0}\right) \Gamma_{0}+o\left(\hat{\mathbf{R}}-\mathbf{R}_{0}\right)
$$

where $\Gamma_{0}$ is the pseudo-inverse of $\left(\mathbf{R}_{0}-\sigma^{2} \mathbf{I}\right)$. By collecting the previous relations, we obtain

$\hat{\tau}_{i}-\tau_{0 i}=\operatorname{Tr}\left[\left(\boldsymbol{\Pi}_{0} \mathbf{D}_{i} \Gamma_{0}+\Gamma_{0} \mathbf{D}_{i} \boldsymbol{\Pi}_{0}\right)\left(\hat{\mathbf{R}}-\mathbf{R}_{0}\right)\right]+o\left(\hat{\mathbf{R}}-\mathbf{R}_{0}\right)$

where

$$
\mathbf{D}_{i} \triangleq \sum_{j=1}^{d}\left[\frac{\partial^{2} \breve{J}}{\partial \tau^{2}}\left(\tau_{0}, \Pi_{0}\right)\right]_{i, j}^{-1} \frac{\partial^{2} \breve{J}}{\partial \tau_{j} \partial \mathbf{\Pi}}\left(\tau_{0}, \mathbf{\Pi}_{0}\right)^{T} .
$$

On the other hand, from (4) and (15), we have

$$
\begin{aligned}
\hat{\mathbf{R}}= & \mathcal{T}_{K}\left(\mathbf{h}_{0}\right) \hat{\mathbf{R}}_{s s} \mathcal{T}_{K}\left(\mathbf{h}_{0}\right)^{H}+\sigma \mathcal{T}_{K}\left(\mathbf{h}_{0}\right) \hat{\mathbf{R}}_{s w} \\
& +\sigma \hat{\mathbf{R}}_{s w}^{H} \mathcal{T}_{K}\left(\mathbf{h}_{0}\right)^{H}+o\left(\sigma^{2}\right)
\end{aligned}
$$

where

$$
\hat{\mathbf{R}}_{s s}=\frac{1}{N-K} \sum_{k=K+1}^{N} \mathbf{s}(k) \mathbf{s}(k)^{H}
$$

and

$$
\hat{\mathbf{R}}_{s w}=\frac{1}{N-K} \sum_{k=K+1}^{N} \mathbf{s}(k) \overrightarrow{\mathbf{n}}(k)^{H} .
$$

As $\boldsymbol{\Pi}_{0} \mathcal{T}_{K}\left(\mathbf{h}_{0}\right)=\mathcal{T}_{K}\left(\mathbf{h}_{0}\right)^{H} \boldsymbol{\Pi}_{0}=0$ and $\boldsymbol{\Pi}_{0} \mathbf{R}_{0}=\sigma^{2} \boldsymbol{\Pi}_{0}$, by keeping only the first order in the noise level, we get

$$
\hat{\tau}_{i}-\tau_{0 i}=\sigma \operatorname{Tr}\left[\hat{\mathbf{R}}_{s w}^{H} \mathbf{M}_{i}+\mathbf{M}_{i}^{H} \hat{\mathbf{R}}_{s w}\right]+o\left(\sigma^{2}\right)+o\left(\mathbf{R}_{0}-\hat{\mathbf{R}}\right)
$$

where $\mathbf{M}_{i} \triangleq \mathcal{T}_{K}\left(\mathbf{h}_{0}\right)^{H} \Gamma_{0} \mathbf{D}_{i} \boldsymbol{\Pi}_{0}$. On the other hand, we need the following lemma. 
Lemma 1: Under assumptions $\mathrm{H} 1$ and $\mathrm{H} 2$, for any arbitrary deterministic matrices $(\mathbf{A}, \mathbf{B})$ in $\mathbb{C}^{K+L+1 \times r(K+1)}$ :

$$
\begin{aligned}
& \lim _{N \rightarrow \infty} N E\left[\operatorname{Tr}\left[\hat{\mathbf{R}}_{s w}^{H} \mathbf{A}\right] \operatorname{Tr}\left[\hat{\mathbf{R}}_{s w}^{H} \mathbf{B}\right]^{*}\right] \\
& =\sum_{k=-(K+1)}^{K+1} \operatorname{Tr}\left[\mathbf{A} \mathbf{J}_{w}(-k) \mathbf{B}^{H} \mathbf{J}_{s}(k)\right] \\
& \lim _{N \rightarrow \infty} N E\left[\operatorname{Tr}\left[\hat{\mathbf{R}}_{s w}^{H} \mathbf{A}\right] \operatorname{Tr}\left[\hat{\mathbf{R}}_{s w}^{H} \mathbf{B}\right]\right]=0
\end{aligned}
$$

where $\mathbf{J}_{w}(k)=\left[\mathbf{J}_{w}(k)_{a b}\right]_{1 \leq a, b \leq r(K+1)}$, and $\mathbf{J}_{s}(k)=$ $\left[\mathbf{J}_{s}(k)_{a b}\right]_{1 \leq a, b \leq K+L+1}$ are two shift matrices defined as $\mathbf{J}_{w}(k)_{a b}=\delta(a-b-r k)$ and $\mathbf{J}_{s}(k)_{a b}=\delta(a-b-k)$. The proof is easy by inspection. Relation (46) follows from the circular property of the noise.

From Lemma 1 and (44), we obtain

$$
\begin{aligned}
& \lim _{N \rightarrow \infty} N E\left[\left(\hat{\tau}_{i}-\tau_{0 i}\right)\left(\hat{\tau}_{j}-\tau_{0 j}\right)\right] \\
& \quad=2 \sigma^{2} \operatorname{Re}\left\{\sum_{k=-(K+1)}^{K+1} \operatorname{Tr}\left[\mathbf{M}_{i} \mathbf{J}_{w}(-k) \mathbf{M}_{j}^{H} \mathbf{J}_{s}(k)\right]\right\}+o\left(\sigma^{2}\right) .
\end{aligned}
$$

Hence, we deduce the expression of the covariance matrix. Moreover, under assumptions $\mathrm{H} 1$ and $\mathrm{H} 2$, the estimated covariance matrix $\hat{\mathbf{R}}_{s w}$ is asymptotically normal with rate of convergence $N^{-1 / 2}$ by an application of the Hoeffding and Robbins theorem for $m$-dependent sequences (see, e.g., Brockwell and Davis [4, Th. 6.4.2, p. 213]). Because the parametric subspace estimator $\hat{\boldsymbol{\tau}}$ is related to $\hat{\mathbf{R}}_{s w}$ through an infinitely differentiable mapping [see (44)], $\hat{\boldsymbol{\tau}}$ is also asymptotically normal with rate $N^{-1 / 2}$.

\section{REFERENCES}

[1] K. Abed-Meraim, J.-F. Cardoso, A. Gorokhov, P. Loubaton, and E. Moulines, "On subspace methods for blind identification of single-input multiple-output fir systems," IEEE Trans. Signal Processing, vol. 45, pp. 42-55, Jan. 1997.

[2] K. Abed-Meraim and Y. Hua, "Blind identification of sparce multipath channels using cyclostationary statistics," in Proc. EUSIPCO, 1998.

[3] D. Astely, B. Ottersten, and A. Swindlehurst, "A generalized array manifold model for local scattering in wireless communications," in Proc. ICASSP, Munich, Germany, Jan. 1997.

[4] P. Brockwell and R. Davis, Time Series: Theory and Methods. New York: Springer, 1990.

[5] M. Cedervall, B. Ng, and A. Paulraj, "Structured methods for blind multi-channel identification," in Proc. ICASSP, July 1997, pp. 387-390.

[6] M. Chenu-Tournier, A. Ferreol, and P. Larzabal, "Low complexity blind space-time identification of propagation parameters," in Proc. ICASSP, Phoenix, AZ, Mar. 1999.

[7] Z. Ding, "Multipath channel identification based on partial system information," IEEE Trans. Signal Processing, vol. 45, pp. 235-240, Jan. 1997.

[8] G. D. Forney, "Minimal bases of rational vector spaces, with applications to multivariable linear systems," SIAM J. Contr., vol. 13, no. 3, pp. 493-520, May 1975.

[9] G. Giannakis, C. Tepedelenlioglu, and H. Liu, "Adaptative blind equalization of time-varying channels," in Proc. ICASSP, 1997.

[10] A. Gorokhov, M. Kristensson, and B. Ottersten, "Robust blind secondorder deconvolution," IEEE Signal Processing Lett., vol. 6, pp. 13-16, Jan. 1999.

[11] J. Grouffaud, P. Larzabal, and H. Clergeot, "Some properties of ordered eigenvalues of a wishart matrix: Application in detection test and model order selection," in Proc. ICASSP, vol. 5, 1996, pp. 2463-2466.

[12] _ "A maximum likelihood approach for passive identification of time-varying multipath channels," in Proc. ICASSP, 1997.
[13] J. K. Hwang and J. H. Chiu, "A new method for blind identification of multipath channel by exploiting signal cyclostationarity," in Proc. SPAWC, 1997, pp. 25-28.

[14] R. Johnson, P. Schniter, T. Endres, J. Behm, D. Brown, and R. Casas, "Blind equalization using the constant modulus criterion: A review," Proc. IEEE, vol. 86, pp. 1927-1950, Oct. 1998.

[15] T. Kailath, Linear Systems. Englewood Cliffs, NJ: Prentice-Hall, 1980.

[16] I. Kang, M. P. Fitz, and S.B. Gelfand, A Novel Approach to the Blind Estimation of Sparce Multipath Channel Parameters, 1997, pp. 80-84.

[17] T. Kato, Perturbation Theory for Linear Operators. New York: Springer-Verlag, 1966.

[18] T. Krauss and M. Zoltowski, "Multiuser blind identification using a linear parametrization of the channel matrix and second order statistics," in Proc. ICASSP, Phoenix, AZ, Mar. 1999.

[19] Y. Li and Z. Ding, "Blind channel identification based on second order cyclostationary statistics," in Proc. ICASSP, vol. 4, 1993, pp. 81-84.

[20] A. Liavas, P. Regalia, and J. P. Delmas, "Robustness of least-squares and subspace methods for blind channel identification/equalization with respect to channel undermodeling," IEEE Trans. Signal Processing, vol. 47, pp. 1636-1645, June 1999.

[21] E. Moulines, P. Duhamel, J.-F. Cardoso, and S. Mayrargue, "Subspace methods for the blind identification of multichannel FIR filters," IEEE Trans. Signal Processing, vol. 43, pp. 516-525, Feb. 1995.

[22] L. Perros-Meilhac, P. Duhamel, P. Chevalier, and E. Moulines, "Blind knowledge based algorithms based on second order statistics," in Proc. ICASSP, Phoenix, AZ, Mar. 1999.

[23] L. Perros-Meilhac and E. Moulines, "Asymptotic performances: Derivations," ENST, Paris, France, Tech. Rep., 1999.

[24] L. Perros-Meilhac, E. Moulines, P. Chevalier, and P. Duhamel, "Identification parametrique et egalisation autodidacte d'un canal speculaire," in Proc. GRETSI, Vannes, France, Sept. 1999.

[25] — "A parametric subspace-based blind estimation of a SIMO-FIR with unknown channel order," in Proc. SPAWC, Annapolis, MD, May 1999.

[26] G. Raleigh and T. Boros, "Joint space-time parameter estimation for wireless communication channels," IEEE Trans. Signal Processing, vol. 46, pp. 1333-1343, May 1998.

[27] S. Schell, D. Smith, and W. Gardner, "Blind channel identification using cyclostationary statistics," in Proc. EUSIPCO, 1994, pp. 716-719.

[28] A. Swindlehurst and J. Gunther, "Methods for blind equalization and resolution of overlapping echoes of unknown shape," IEEE Trans. Signal Processing, vol. 47, pp. 1245-1254, May 1999.

[29] L. Tong and S. Perreau, "Multichannel blind identification: From subspace to maximum likelihood methods," Proc. IEEE, vol. 86, pp. 1951-1967, Oct. 1998.

[30] L. Tong, G. Xu, and T. Kailath, "A new approach to blind identification and equalization of multipath channels," in Proc. 25th Asilomar Conf. Signals, Syst., Comput., Pacific Grove, CA, 1991, pp. 856-860.

[31] A. van der Veen, M. Vanderveen, and A. Paulraj, "Joint angle and delay estimation using shift-invariance techniques," IEEE Trans. Signal Processing, vol. 46, pp. 405-418, Feb. 1998.

[32] M. Vanderveen, A. van der Veen, and A. Paulraj, "Estimation of multipath parameters in wireless communications," IEEE Trans. Signal Processing, vol. 46, pp. 682-690, Mar. 1998.

[33] M. C. Vanderveen and A. Paulraj, "Improved blind channel identification using a parametric approach," IEEE Commun. Lett., pp. 226-228, Aug. 1998.

[34] G. Venkatesan, L. Tong, M. Kaveh, A. Tewfik, and K. Buckley, "A deterministic blind identification technique for simo systems of unknown model order," in Proc. ICASSP, Phoenix, AZ, Mar. 1999.

[35] M. Wax and T. Kailath, "Detection of signals by information theoric criteria," IEEE Trans. Acoust. Speech, Signal Processing, vol. ASSP-33, pp. 387-392, Apr. 1985.

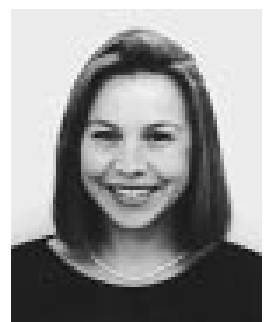

Lisa Perros-Meilhac was born in Cagnes sur mer, France, in 1975. She received the M.Sc. degree in electrical engineering and the Postgraduate degree in digital communication systems from Ecole Nationale Superieure des Telecommunications (ENST), Paris, France in 1997. Since October 1997, she has been pursuing the Ph.D. degree with the Image and Signal Processing Department, ENST, with support from CNRS and Thomson-CSF Communications.

Her research concerns blind array signal processing for digital communications. 
Éric Moulines (M'91) was born in Bordeaux, France, in 1963. He received the M.S. degree from Ecole Polytechnique, Paris, France, in 1984 and the Ph.D. degree from Ecole Nationale Superieure des Telecommunications (ENST), Paris, in signal processing in 1990.

From 1986 until 1990, he was Member of the Technical Staff at CNET, working on signal processing applied to low-bit rate speech coding and text-to-speech synthesis. Since 1990, he has been with ENST, where he is presently a Professor. His teaching and research interests include statistical signal processing and speech processing. Currently, he is engaged in research in various aspects of statistical signal processing including, among others, single and multichannel ARMA filtering and modeling, blind signal processing for digital communications, characterization and estimation of point processes with application to high bit-rate data traffic modeling, low bit-rate speech coding, and speech transformation. He is an Associate Editor of Speech Communication.

Dr. Moulines is an Associate Editor the IEEE TRANSACTIONS ON SIGNAL PROCESSING. He is a Member of the IEEE Committees on Speech and Statistical Signal and Array Processing.

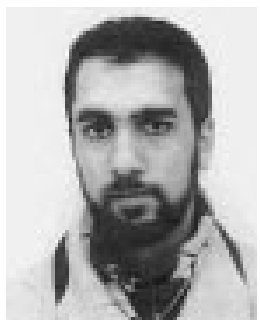

Karim Abed-Meraim was born in Algiers, Algeria, in 1967. He received the M.S. degree from Ecole Polytechnique, Paris, France, in 1990 and the Ph.D. degree from Ecole Nationale Superieure des Telecommunications (ENST), Paris, in 1995 in the field of signal processing and communications.

He currently is Associate Professor at the Signal and Image Processing Department, ENST. His research interests include statistical signal processing, system identification, multiuser detection, space-time coding, (blind) array processing, and

performance analysis.

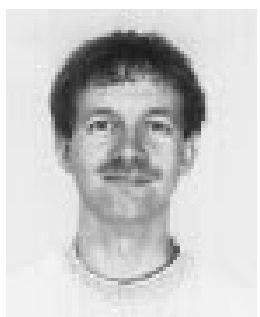

Pascal Chevalier was born in 1962 in Valenciennes, France. He received the M.Sc. degree from Ecole Nationale Suprieure des Techniques Avances (ENSTA), Paris, France, and the Ph.D. degree from University of Paris-Sud in 1985 and 1991, respectively.

Since 1991, he has been in industry (studies, experimentations, expertise, management), teaching activities, both in French engineering schools (Supelec, ENSTA) and French Universities (Cergy-Pontoise), and research activities. His present research interests are in array processing techniques, either blind or informed, second- or higher order, spatial-or spatio-temporal, time-invariant or time-varying especially for cyclostationary signals, linear or nonlinear and, particularly, widely linear for noncircular signals, for applications such as TDMA and CDMA radiocommunications networks, satellite telecommunications, spectrum monitoring, and HF/VUHF passive listening. He is author or co-author of more than 50 papers in journals and conferences, eight patents, and several book chapters.

Dr Chevalier is a member of the THOMSON-CSF Technical and Scientific Council and of EURASIP and is a senior member of the Societ des Electriciens et des Electroniciens (SEE).

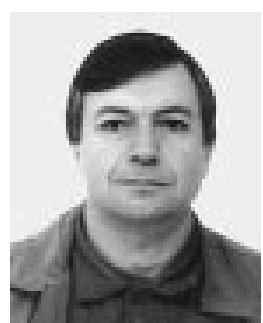

Pierre Duhamel (S'87-F'98) was born in France in 1953. He received the Ing. degree in electrical engineering from the National Institute for Applied Sciences (INSA), Rennes, France, in 1975 and the Dr.Ing. degree in 1978 and the Dr.Sci. degree in 1986, both from Orsay University, Orsay, France.

From 1975 to 1980, he was with Thomson-CSF, Paris, France, where his research interests were in circuit theory and signal processing, including digital filtering and analog fault diagnosis. In 1980, he joined the National Research Center in Telecommunications (CNET), Issy les Moulineaux, France, where his research activities were first concerned with the design of recursive CCD filters. Later, he worked on fast Fourier transforms and convolution algorithms and applied similar techniques to adaptive filtering, spectral analysis, and wavelet transforms. From 1993 to September 2000, he was a Professor at Ecole Nationale Superieure des Telecommunications (ENST), Paris, with research activities focused on signal processing for communications. He was head of the Signal and Image Processing Department from 1997 to 2000. He is now with the Laboratoire de Signaux et Systemes, Gif sur Yvette, France, where he is developing studies in signal processing for communications (including equalization, iterative decoding, and multicarrier systems) and signal/image processing for multimedia applications, including source coding-image coding, multichannel sound coding, joint source/channel coding, watermarking, and audio processing.

Dr. Duhamel was chairman of the DSP committee of the IEE Signal Processing Society from 1996 to 1998, was an Associate Editor of the IEEE TRANSACTIONS ON SIGNAL PROCESSING from 1989 to 1991, and was Associate Editor for the IEEE Signal Processing LeTters. He was a Guest Editor for the special issue of the IEEE TRANSACTIONS ON Signal PROCESSING on wavelets. He is now a member of the SP for Communications Committee. He was an IEEE Distinguished Lecturer for 1999 and is co-general chair of the 2001 International Workshop on Multimedia Signal Processing, Cannes, France. The paper on subspace-based methods for blind equalization, which he co-authored, received the "Best paper award" from the IEEE TRANSACTIONS ON Signal PROCESSING in 1998. He was awarded the "Grand Prix France Telecom" by the French Science Academy in 2000. 